\title{
Robust optimization of sustainable food grain transportation with uncertain supply and intentional disruptions
}

\begin{abstract}
The proliferating need for sustainability intervention in food grain transportation planning is anchoring the attention of researchers in the interests of stakeholders and environment at large. Uncertainty associated with food grain supply further intensifies the problem steering the need for designing robust, cost-efficient and sustainable models. In line with this, this paper aims to develop a robust and sustainable intermodal transportation model to facilitate single type of commodity food grain shipments while considering procurement uncertainty, greenhouse gas (GHG) emissions, and intentional hub disruption. The problem is designed as a mixed integer non-linear robust optimization model on a hub and spoke network for evaluating near optimal shipment quantity, route selection and hub location decisions. The robust optimization approach considers minimization of total relative regret associated with total cost subject to several real time constraints. A version of Particle Swarm Optimization with Differential Evolution (PSODE) is proposed to tackle the resulting NP-hard problem. The model is tested with two other state-of the art meta-heuristics for small, medium, and large datasets subject to different procurement scenarios inspired from real time food grain operations in Indian context. Finally, the solution is evaluated with respect to total cost, model and solution robustness for all instances.
\end{abstract}

Keywords: Transportation; Sustainable supply chain; Robust optimization; Particle swarm optimization; Metaheuristics 


\section{Introduction}

According to global statistics put forth by Food and Agriculture organization (FAO), the annual wastage of food grains is 1.3 billion tons, and up to $20 \%$ of annual food grain produce are wasted in the post-harvest phases of developing countries (Zorya et al. 2011). Major reasons for witnessing the heavy magnitude of losses in Indian context have been attributed to improper utilization of government infrastructure and resources, uncertainty associated with food grain supply, and untimely planning of rake allocation and scheduling (CAG report 2013). In addition, food grain consumption levels in India are reportedly less as compared to the world due to shortage of the staple food available for direct intake (CAG report 2013). The food grain demand is almost constant and is extrapolated from population census data, whereas the abundance and uncertainty associated with the food grain yield poses numerous challenges. Balancing the mismatch between uncertain supply and deterministic demand points with efficient utilization of various resources is a striking challenge in Indian context owing to geographically widespread yields. Given the huge financial outlay (Rs 90000 crore) of ongoing food grain operations (Narayanan 2015) and the severity of the aforementioned issues, the need for particular emphasis on streamlining Indian food grain supply chain with focus to address supply uncertainty is an immediate necessity.

In addition to the aforementioned concerns, rapidly increasing levels of global warming, advancement of environmental regulations and higher rates of natural calamities, compel the need for holistic design of food grain supply chain networks by incorporating sustainability. Thirty eight percent of global GHG emissions is attributed to transportation and agriculture sectors (IPCC 2014). Multimodal freight transportation, shipping operations, carbon intensive manufacturing activities, and intermodal facilities are prime sources of GHG emissions where the implementation of carbon sensitive design of supply networks is of utmost importance. Over 35 million tons of 
food grains are transported annually through railways and roadways, thus significantly contributing to freight GHG emissions (fci.gov.in/movements.php). Intermodal and multimodal freight transportation, shipping operations, carbon intensive manufacturing activities, and intermodal facilities are prime sources of GHG emissions. Food and Agriculture organization points out that a kilogram of food lost in the later stages of supply chain (post harvest stages) possess higher carbon intensity than a kilogram lost at preliminary stages. In India, agriculture contributes to $18 \%$ of total GHG emissions (INCCA 2010). Food grains alone accounted for 7,900 kilograms of $\mathrm{CO}_{2}$ emissions in the subcontinent in 2003 (Parikh et al. 2009). The forecasts by Indian Council of Agricultural Research (ICAR) suggest that the area-averaged annual mean warming is estimated to range between $1{ }^{\circ} \mathrm{C}-4{ }^{\circ} \mathrm{C}$ by 2020 and between $2.2^{\circ} \mathrm{C}-2.9^{\circ} \mathrm{C}$ by 2050 . Thus, the need for considering environmental issues in the design of food grain transportation systems, with focus to reduce emissions is justified.

Apart from environmental concerns, the food grain supply chain network design also holds the need for simultaneous focus to curb the impact of disruption. The adverse impact of floods at West Bengal, India in 2016, and the infamous Bhuj earthquake in Gujrat provide a wakeup call and testify the need for focusing on disruption to safeguard the population against staple food. This draws the attention of researchers to accommodate design for disruptions in food grain supply chain. According to Parvaresh et al. (2014), supply chain design for natural calamities, terrorists attacks and shutdowns have been modelled as intentional disruption. The possibility of maintaining adaptive capacity for dealing with disruption was recognized by Williams et al. (2017) who emphasized the importance of extending the step forward towards inculcating sustainability. However, the literature pertaining to collective design for sustainability, supply uncertainty and intentional disruptions is underdeveloped and attracts deeper attention. 
Based on the aforementioned concerns, this research explores the idea of integrating economic and environmental sustainability with food grain transportation network design subject to uncertain supply and intentional disruptions. . It is built upon the framework proposed by Maiyar et al. (2015) for food grain supply chain system in Indian context. The proposed model in this paper facilitates interstate transportation in between the central level warehouses of two different states. Robust optimization approach is adopted to deal with uncertainty associated with food grain supply. The proposed model is ensured to be model robust and solution robust by enforcing penalty on control constraints and considering the minimization of total relative regret associated with total cost respectively. The total cost captures total shipment costs from warehouses in origin state to warehouses in destination states through origin and destination intermodal hubs, facility location costs, rerouting costs and GHG emissions costs. The constraints of the problem ensure to satisfy demand, warehouse capacity, vehicle capacity, hub allocation, emergency hub and flow balance requirements. The resulting mixed integer non-linear robust optimization model is solved using self-tailored particle swarm optimization with differential evolution (PSODE), particle swarm optimization (PSO) and firefly algorithm (FFA). Finally, the robustness of the solutions obtained are verified with respect to model robustness and solution robustness for small, medium and large size data sets.

The subsequent sections are organized as follows. The second section highlights the literature review conducted and presents the identification of research gaps. Section 3 provides the problem description with figurative illustration. Section 4 delineates the proposed mathematical model. Section 5 describes the tailoring of hybrid particle swarm optimization with differential evolution algorithm followed by the plan of experiments in Section 6. The explanation of result and detailed 
discussions are carried in Section 7. Lastly, section 8 provides conclusion, limitation and future scope.

\section{Literature review}

The following systematic approach was employed for conducting literature review. Initially, the keywords "supply chain network design with uncertainty", "supply chain network design with disruption" and "sustainable supply chain design" were used to identify the relevant papers from the web of science and scopus database. Later on, the literature concerning to supply chain network design was reviewed to understand present contributions and future direction. During the review process, the review paper authored by Govindan et al. (2017) was identified as a reference to further narrow down the focus for identifying problems which addressed uncertainty, sustainability and disruption mathematically. The literature pertaining to uncertainty modelling in supply chain network design can be broadly classified into various categories of problems based on modelling approach, problem environment and solution methodology as shown in Figure 1. Based on the uncertainty source, the risks associated with supply chain design are categorized as operational and disruption risks (Govindan et al. 2017). Disruption risks can be intentional (war and terrorist attacks) or unintentional (floods, natural disasters etc.). The uncertainty arising from unintentional disruptions are captured by assuming discrete or continues probability scenarios whereas intentional disruptions are modelled as discrete problems (Parvaresh et al. 2014). Blackhurst et al. (2005) emphasized the need for real time supply chain reconfiguration and reachability analysis to deal with disruption recovery issues in supply chain network. In this regard, they discussed the importance of robust modelling approach towards the design of resilient supply chain network. According to Chopra and Sodhi (2004), a disruption in supply chain due to natural disaster or terrorism significantly affects the overall cost. Wagner and Bode (2008) conducted an empirical 
investigation that confirmed the adverse effect of supply side risk and risks from catastrophes on supply chain performance. The present work gains a unique position in this framework of existing literature by its contribution towards the development of a non-linear and multi-period robust optimization model characterized by discrete supply side uncertainty and intentional hub disruptions. The aforesaid model is designed to minimize total costs associated with transportation, facility location, rerouting and GHG emissions. Table 1 illustrates the different methodologies adopted for uncertainty modelling in supply chain network design and the convergence or divergence of the present methodology from standard approaches with respect to various modelling intricacies. The proposed hybrid approach combines the robust optimization approach adopted by Rosa et al. (2013) with particle swarm optimization and differential evolution algorithm (PSODE) to arrive at near optimal solutions. Owing to the higher capability of PSODE in striking the balance between exploration and exploitation of the search space (Epitropakis et al. 2012), as different from the previously developed hybrid approaches, the current hybrid approach guarantees robust as well as higher quality of near optimal solution.

It is important to understand the conceptualization of robust optimization put forth by several authors to deal with uncertainty (Laguna 1998). Robust models under polyhedral uncertainty sets have been extensively studied and implemented on linear problems (Jalilvand-Nejad et al. 2016; Bertsimas and Sim 2004) and later extended to address mixed integer programming problems (Liu et al. 2018). The proofs derived by Bertsimas and Sim (2004) for robust counterparts under polyhedral uncertainties cannot be generalized for non-linear mixed integer formulations. Therefore, Ben-Tal et al. (2015) proposed a robust counterpart for non-linear uncertainties which fall within the boundary of convex programming problems. 


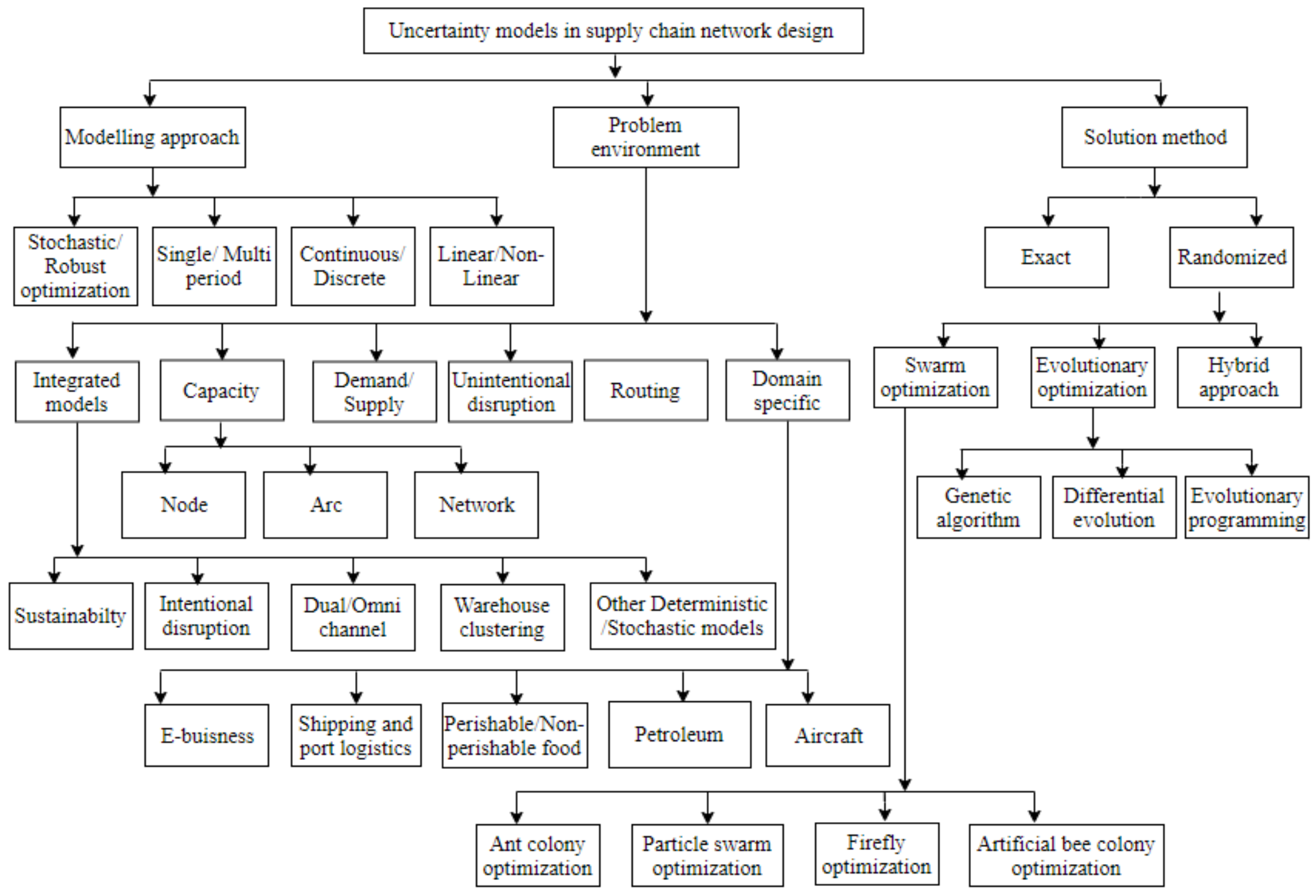

Figure 1. Hierarchical classification of uncertainty literature pertaining to supply chain network design. 
Table1. Methodology comparison study of relevant literature with present work

\begin{tabular}{|c|c|c|c|c|c|c|c|c|c|c|}
\hline $\begin{array}{l}\text { Literature } \\
\text { study }\end{array}$ & Methodology & Linear & $\begin{array}{l}\text { Mixed } \\
\text { integer } \\
\text { linear }\end{array}$ & $\begin{array}{l}\text { Non- } \\
\text { linear } \\
\text { and } \\
\text { convex }\end{array}$ & $\begin{array}{l}\text { Non- } \\
\text { linear } \\
\text { and non- } \\
\text { convex }\end{array}$ & $\begin{array}{l}\text { Mixed } \\
\text { integer } \\
\text { non-linear } \\
\text { and convex }\end{array}$ & $\begin{array}{l}\text { Mixed } \\
\text { integer non- } \\
\text { linear and } \\
\text { non-convex }\end{array}$ & Exploration & Exploitation & Optimality \\
\hline $\begin{array}{l}\text { Khatami et al. } \\
(2015)\end{array}$ & Decomposition & $\checkmark$ & $\checkmark$ & $x$ & $x$ & $x$ & $x$ & $\checkmark$ & $\checkmark$ & Optimum \\
\hline $\begin{array}{l}\text { Shu et al. } \\
\text { (2010) }\end{array}$ & $\begin{array}{l}\text { Column generation and } \\
\text { Stochastic optimization } \\
\text { (SO) }\end{array}$ & $\checkmark$ & $\checkmark$ & $\checkmark$ & $x$ & $\checkmark$ & $x$ & $\checkmark$ & $\checkmark$ & Optimum \\
\hline $\begin{array}{l}\text { Azizi et al. } \\
(2016)\end{array}$ & Genetic Algorithm(GA) & $\checkmark$ & $\checkmark$ & $\checkmark$ & $\checkmark$ & $\checkmark$ & $\checkmark$ & $\checkmark$ & $x$ & $\begin{array}{l}\text { Near- } \\
\text { optimum }\end{array}$ \\
\hline $\begin{array}{l}\text { Hachicha et al. } \\
\text { (2011) }\end{array}$ & $\begin{array}{l}\text { Differential Evolution } \\
\text { (DE) }\end{array}$ & $\checkmark$ & $\checkmark$ & $\checkmark$ & $\checkmark$ & $\checkmark$ & $\checkmark$ & $\checkmark$ & $x$ & $\begin{array}{l}\text { Near- } \\
\text { optimum }\end{array}$ \\
\hline $\begin{array}{l}\text { Bozorgi- } \\
\text { Amiri et al. } \\
(2012)\end{array}$ & $\begin{array}{l}\text { Particle Swarm } \\
\text { optimization (PSO) }\end{array}$ & $\checkmark$ & $\checkmark$ & $\checkmark$ & $\checkmark$ & $\checkmark$ & $\checkmark$ & $x$ & $\checkmark$ & $\begin{array}{l}\text { Near- } \\
\text { optimum }\end{array}$ \\
\hline $\begin{array}{l}\text { Huang and } \\
\text { Lin }(2010)\end{array}$ & $\begin{array}{l}\text { Ant Colony optimization } \\
\text { (ACO) }\end{array}$ & $\checkmark$ & $\checkmark$ & $\checkmark$ & $\checkmark$ & $\checkmark$ & $\checkmark$ & $x$ & $\checkmark$ & $\begin{array}{l}\text { Near- } \\
\text { optimum }\end{array}$ \\
\hline $\begin{array}{l}\text { Nickel et al. } \\
\text { (2012) }\end{array}$ & Commercial solvers & $\checkmark$ & $\checkmark$ & $\checkmark$ & $x$ & $\checkmark$ & $x$ & $\checkmark$ & $\checkmark$ & Optimum \\
\hline $\begin{array}{l}\text { Rosa et al. } \\
\text { (2013) }\end{array}$ & $\begin{array}{l}\text { Robust optimization } \\
\text { with discrete scenarios } \\
\text { (ROD) }\end{array}$ & $\checkmark$ & $\checkmark$ & $\checkmark$ & $\checkmark$ & $\checkmark$ & $\checkmark$ & - & - & $\begin{array}{l}\text { Robust } \\
\text { near } \\
\text { optimum }\end{array}$ \\
\hline $\begin{array}{l}\text { Ben-Tal et al. } \\
(2015)\end{array}$ & $\begin{array}{l}\text { Robust optimization } \\
\text { with interval sets (ROI) }\end{array}$ & $\checkmark$ & $\checkmark$ & $\checkmark$ & $x$ & $x$ & $x$ & - & - & $\begin{array}{l}\text { Robust } \\
\text { optimum }\end{array}$ \\
\hline $\begin{array}{l}\text { Jung et al. } \\
\text { (2004) }\end{array}$ & Simulation & $\checkmark$ & $\checkmark$ & $\checkmark$ & $\checkmark$ & $\checkmark$ & $\checkmark$ & - & - & $\begin{array}{l}\text { Sub } \\
\text { optimum }\end{array}$ \\
\hline Present study & RO based PSODE & $\checkmark$ & $\checkmark$ & $\checkmark$ & $\checkmark$ & $\checkmark$ & $\checkmark$ & $\checkmark$ & $\checkmark$ & $\begin{array}{l}\text { Near- } \\
\text { optimum }\end{array}$ \\
\hline
\end{tabular}


However, any such method containing polyhedral sets with uncertain parameters has not yet been developed for problems which are of non-convex type. Mulvey et al. (1995) pioneered the concept of solution and model robustness while illustrating the desirable properties of a solution to robust optimization models. They developed models for several practical applications including power capacity expansion, matrix balancing and image reconstruction, scenario immunization for financial planning, air-force airline scheduling; and minimum weight structure design while capturing the trade-offs between two type of robustness. Rahmani et al. (2013) developed a robust optimization model which aims at minimizing the total cost and decreasing the influence of uncertainty in the production costs and demand for multiple products in dual stage production. Amin and Zhang (2013) recommended a multi-objective facility location model that investigates the influence of uncertainty of demand and return on a closed-loop supply chain network. Govindan et al. (2015) developed a multi-objective optimization model that emphasizes to minimize the total costs and ecological effect of combining supply chain network design and order allocation problem. Lium et al. (2009) examined the significant role of demand stochasticity in service network design. Zeballos et al. (2014) developed a design and planning approach to assess the impact of uncertain customer demand and supply of raw material on network considering multi-period and multi-product closed-loop supply chains scenario. Rosa et al. (2013) proposed a robust capacitated facility location model which aims at minimizing the expectations of relative regrets for a number of scenarios considering uncertain supply and demand of goods. Hasany and Shafahi (2017) suggested a two-stage stochastic program that determines optimal shipment paths while addressing the railroad blocking problem under uncertain demand and supply of resource. Govindan et al. (2017) described the applicability, advantages and disadvantages of various 
uncertainty handling approaches such as risk-averse stochastic programming, recourse-based stochastic programming, fuzzy mathematical programming, and robust optimization.

There is paucity of literature that highlights sustainability and uncertainty issues in food supply chains. The empirical study conducted by Mangla et al. (2018) employs interpretive structural modelling technique to identify the contextual relationship and relative importance of dependent and independent enablers that drive sustainable initiatives in Indian context. They confirm that focussing on resources allocation and information sharing, capacity building, supply chain monitoring, developing competitive advantages, cost effectiveness and performance improvement are important dependent enablers that significantly contribute to sustainable development. They also confirm that the impact of these enablers can be realized through the implementation of independent enablers such as governmental and non-governmental pressure, regulatory interference, incentives for sustainability implementation, customer and stakeholder understanding, sustainability awareness, and management support. While the mathematical treatment and analysis of independent variables is beyond the scope of this research, the present study aims to quantitatively capture dependent variables such as resource allocation, capacity building, cost effectiveness, and performance improvement. In the context of quantitative modeling, Ahumada et al. (2012) presented a stochastic tactical planning model that incorporates uncertainty encountered in the fresh produce industry in order to develop robust growing plans. Bortolini et al. (2016) introduced a three-objective linear programming model to handle tactical optimization pertaining to fresh food distribution networks comprising of operating cost, carbon footprint and delivery time goals. Borodin et al. (2016) provided an overview of operations research methodologies which deal with uncertainty in the agri-based supply chain management. Garrone et al. (2014) provided a multi-dimensional concept of sustainability in food supply chain 
by offering Availability-Surplus-Recoverability-Waste (ASRW) approach, which integrated the food supply chain with environmental, enterprise and social participants. Akkerman et al. (2010) reviewed quantitative methodologies to food distribution management, and identified challenges encountered by the food industry. They investigate the importance of food safety, food quality and sustainability at strategic, tactical, and operational decision levels of transportation planning. Accorsi et al. (2016) presented a framework to support strategic decision-making that addresses the problems pertaining to agriculture and food distribution considering climate stability. The authors aimed to resolve an original agro food land-network problem with localized as well as large-scaled decisions for optimal land-use allocation and location-allocation.

While the rigorous mathematical treatment of food grain transportation planning with sustainability and uncertainty concerns has been conducted independently in literature, efforts to visualize and implement an integrated approach considering both the issues is scarcely seen and often neglected in this domain. Ge et al. (2015) dealt with quality control issues subject to uncertainty with respect to delivery of eligible wheat in the context of Canadian wheat supply chain. Focusing on sustainability, Maiyar and Thakkar (In press) developed a food grain transportation model considering social and environmental costs in the presence of intentional disruptions while ignoring uncertainty issues. Barbosa-Póvoa et al. (2018) reviewed the trends and directions of operation research methods and their applications towards the achievement of sustainable supply chain. Garnett (2011) conducted a review based study estimating the greenhouse gas (GHG) emissions from foods at the regional, national and global levels, highlighting both GHG-intensive stages and GHG-intensive food types in food chains. Wakeland et al. (2012) described different methods for evaluating the energy intensity and emissions from a food distribution system, assessing various trade-offs between facets of sustainability (economic, 
environmental and equity) and identifying improvement opportunities. Maiyar and Thakkar (2019) presented a multi-objective model to minimize GHG emissions and total supply network cost considering food grain wastages, while they ignored the impact of uncertain supply. Zhu et al. (2018) published a model based review summarizing the recent advancements and opportunities for sustainable modelling of food supply chains considering economic, environmental and social perspectives. Focusing on disruptions, Diabat et al. (2012), proposed a risk averse interpretive structural model (ISM) considering five types of risks pertaining to food industry. Huang et al. (2018) dealt with pricing and inventory decisions with production disruption and governable deterioration for food supply chain.

The aforementioned review of the existing literature reveals that there is huge potential and need for developing integrated and robust transportation models that capture sustainability and uncertainty in food grain supply chains. Thus, the current paper attempts to bridge this gap and attempts to contribute to the existing literature in the following specific aspects. Firstly, it formulates a mixed integer non-linear robust optimization model with focus to uniquely capture supply uncertainty, design for disruptions and environmental concerns simultaneously in Indian food grain context. Secondly, the paper contributes to transportation literature by considering, hub allocation, vehicle capacity, warehouse capacity and emergency hub constraints while realizing the shipments in intermodal setting on hub and spoke system. Thirdly, a hybrid approach which combines the robust optimization approach adopted by Rosa et al. (2013) with particle swarm optimization and differential evolution algorithm (PSODE) to arrive at near optimal solutions is proposed. The detailed problem, model formulation and solution approach are delineated in further parts of this study. 


\section{Problem environment}

This paper addresses the transportation problem invigorated from Indian food grain supply chain network connecting two states, Andhra Pradesh and Tamil Nadu, hailing from southern region of India. Every state in the subcontinent is demographically divided into a number of regions. In the current practice, the food grain system undertakes two types of procurement: Centralized and Decentralized. In decentralized procurement, food grains are procured by the state government authorities whereas in the later case the central government agency (Food Corporation of India) undertakes procurement of food grains. Food grains procured through decentralized procurement strategy are stored in warehouses owned or hired by the state and are used to satisfy the demand within the state. On the other hand, food grains procured through centralized procurement strategy are stored in warehouses owned or hired by the central government and are used to satisfy the demand of another state. The focus of this work aligns within the operational spectrum of centralized procurement, which is undertaken by Food Corporation of India (FCI), a central government agency that is responsible for procurement, storage and transportation of food grains. Every region inside a particular state is further divided into limited number of warehouses. A fixed number of such warehouses are positioned as potential intermodal transport hubs from where the incoming flow of food grains from trucks are transferred to trains, which are subsequently shipped by rail to the destination state. The food grain warehouses present in each region are segregated into two types: (1) intermodal hub warehouse and (2) non-hub warehouse. Intermodal hub warehouses are categorized as the warehouses which have the capacity to handle intermodal transfer operations in addition to storage of food grains, whereas non-hub warehouse are described as the ones that are employed for only storing the food grains. The food grains to be transferred either originate from a non-hub warehouse or from an intermodal hub warehouse of the origin 
(surplus) state and continue towards the designated non-hub warehouse or intermodal hub warehouse of the destination (deficit) state. Since, the interstate transportation takes place only through rail, the non-hub warehouses are not equipped with rail road facilities to receive the stock of food grains from another state. The set of routes therefore between the states is nearly fixed and can be altered only with government intervention or change of interstate wagon management policies. Therefore, direct shipments from origin to destination non-hub warehouses are not allowed and are transported only through intermodal hubs. The two types of warehouses within a state are linked either by road or rail. Figure 2 shows the pictorial outline of the supply network with the intermodal links connecting distinctive nodal elements pertaining to the problem while it illustrates the flow of food grains from origin warehouse (OW) to destination warehouse (DW) for different cases of disruption. The dotted unidirectional arrow that corresponds to "disruption at only destination hub" in Figure 2, represents the flow of food grains from OW to DW for the case when disruption occurs only in one of the destination intermodal hubs. In such a case, there is no disruption of origin hubs. For example, if there are 3 potential origin hubs and all the origin hubs are selected to be opened, then the total number of disrupted hubs in the origin state would be zero, and the flow shall not be routed through origin emergency hubs and will be routed through one of the three selected origin intermodal hubs $(\mathrm{OIH})$. Whereas, since the destination intermodal hubs (DIH) are disrupted in this case, the disrupted flow is rerouted through the emergency destination intermodal hub as shown in Figure 2. Therefore, for the above scenario, the food grain flow from origin warehouse $(\mathrm{OW})$ to destination warehouse (DW), passes through origin intermodal hubs $(\mathrm{OIH})$ and destination emergency hubs (DEH). 


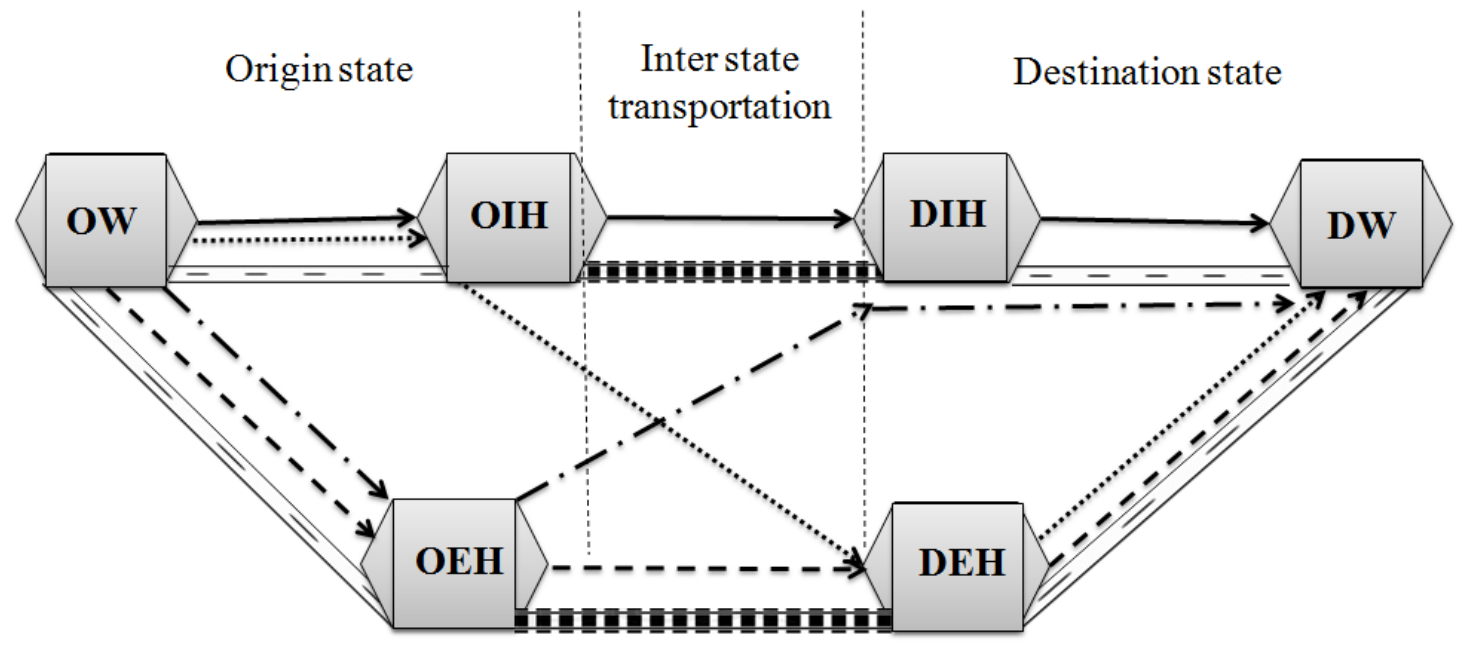

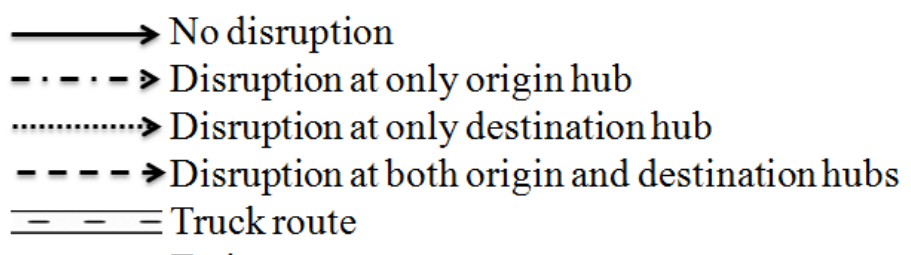

믈를르를믈를 Train route
OW: Origin warehouse OIH: Origin intermodal hub OEH: Origin emergency hub DIH: Destination intermodal hub DEH: Destination emergency hub DW: Destination warehouse

Figure 2. Outline of food grain supply network

As stated earlier in section 1, the current scenario of food grain procurement in Indian context is highly randomized with abundant and uncertain yield. As a consequence, it becomes highly challenging to capture the nature of probability distribution that exactly defines the procurement patterns. Hence, in this paper, we choose to represent the situation of supply uncertainty as a discrete equivalent optimization problem considering different scenarios of food grain procurement denoted by $s \in S$, where $S$ is the set of all scenarios, associated with probability of occurrence $p_{s}$ for each scenario $s$ such that, $\sum_{s \in S} p_{s}=1$.

\section{Mathematical model}

As different from the robust optimization models with uncertainty sets proposed by Bertsimas and Sim (2004) and traditional stochastic programming approaches (risk-averse stochastic 
programming, recourse-based stochastic programming, fuzzy mathematical programming, etc.), the sustainable and stochastic intermodal food grain transportation problem addressed in this work is articulated in accordance with the robust design for stochastic optimization proposed by Rosa et al. (2013). The later approach is line with the conceptualization put forth by Mulvey et al. (1995) who highlighted the importance of robust formulations considering the trade-off between model robustness and solution robustness for a wide class of linear as well as non-linear problems subject to erroneous or uncertain parameters. Therefore, this method of uncertainty modelling is adopted in this work to tackle with the resulting non-linear and NP hard nature of the formulation. In the present problem, the inclusion of penalty for infeasibilities occurring from uncertain realization of decision variables accounts for model robustness, whereas, solution robustness is ensured by considering minimization of total relative regret associated with the total costs. A clear illustration of the proposed robust optimization framework is shown in Figure 3.

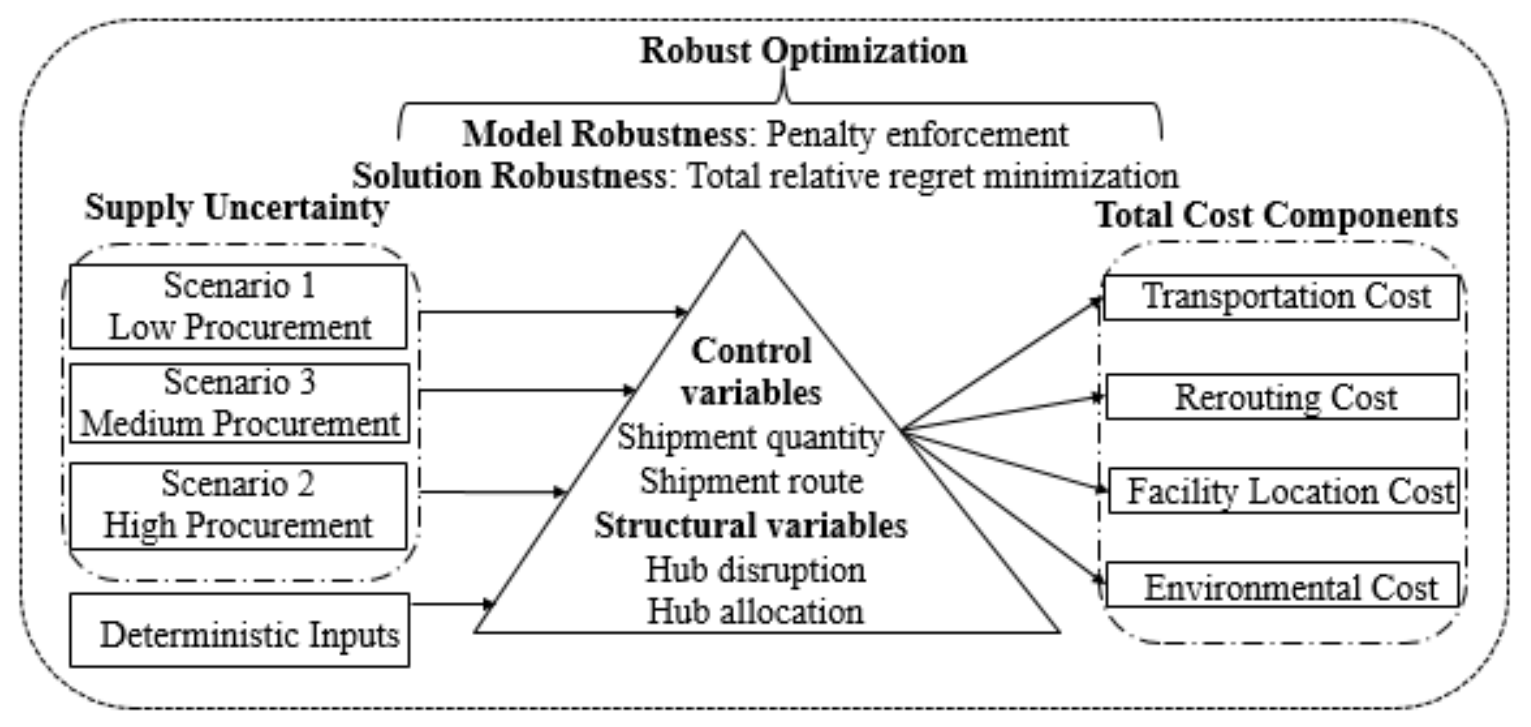

Figure 3. Robust optimization framework

The following assumptions are required for mathematical conceptualization of the practical large scale stochastic problem addressed in this work: 
- Single type of food grain is transported

- Nature of demand is known and deterministic

- Vehicles carry Full Truck Load (FTL) transport

- In each region one warehouse is at least present.

- In each state one hub is at least open

- Emergency hubs are not disrupted

- The shipment is completed in one time period

- Supply uncertainty is unknown and discrete

Table 2. Table of indices and sets

\begin{tabular}{|c|c|c|c|}
\hline Symbol & Description & Set & Definition \\
\hline$i$ & $\begin{array}{l}\text { Origin FCI } \\
\text { warehouse }\end{array}$ & $R_{o}$ & Regions in origin state $o$ \\
\hline$j$ & $\begin{array}{l}\text { Destination FCI } \\
\text { warehouse }\end{array}$ & $R_{d}$ & Regions in destination state $d$ \\
\hline$k$ & Origin hub & $W_{p}$ & Warehouses in origin region $p$ \\
\hline$m$ & Destination hub & $W_{o}$ & Warehouse in origin state, $W_{o}=\bigcup W_{p}$ \\
\hline$e$ & $\begin{array}{l}\text { Origin } \\
\text { emergency hub }\end{array}$ & $W_{q}$ & Warehouses in destination region $q$ \\
\hline$f$ & $\begin{array}{l}\text { Destination } \\
\text { emergency hub }\end{array}$ & $W_{d}$ & Warehouses in destination state, $W_{d}=\bigcup_{q=1}^{\left|R_{d}\right|} W_{q}$ \\
\hline$\phi$ & Road & $H_{o}$ & Potential hub locations in origin state $o, H_{o} \subset \bigcup_{p=1}^{\Lambda_{o} \mid} W_{p}$ \\
\hline$\psi$ & Rail/Rake & $H_{d}$ & $\begin{array}{l}\text { Set of potential hub locations in destination state } d \text {, } \\
H_{d} \subset \bigcup^{\left|R_{d}\right|} W_{q}\end{array}$ \\
\hline$t$ & Time period & $T$ & Time periods \\
\hline$s$ & Scenario & $S$ & Discrete scenarios \\
\hline
\end{tabular}


The list of definitions for indices and sets followed while formalizing the stochastic problem with uncertain supply are shown in Table 2 . The information regarding potential hubs is known prior to start of planning process. They are mathematically represented as a subset of origin or destination warehouses. The advantage of flow consolidation at the intermodal hubs is quantitatively captured by using cost consolidation factor $(\alpha \mid(0<\alpha<1))$. The unconsolidated unit transportation cost through intermodal hubs $k\left(k \in H_{o}\right)$ and $m\left(m \in H_{d}\right)$, is $T R_{k m}$. Given this, actual hub to hub unit transportation cost is estimated as, $\alpha\left(T R_{k m}\right)$, where, $1-\alpha$ denotes the fractional reduction in the unconsolidated price caused by economies of scale associated with consolidation.

$C T_{i k m j}=R O_{i k}+\alpha T R_{k m}+R D_{m j}, \forall i, \forall j, \forall k, \forall m$

A linear cost function as presented in Equation (1) is employed to estimate intermodal transportation cost for one unit of food grain from warehouse $i\left(i \in W_{o}\right)$ to warehouse $j\left(j \in W_{d}\right)$

. Here, the unit transportation cost, $C T_{i k m j}$ is the summation of three components. The first component is the unit cost of transportation by road from warehouse $i\left(i \in W_{o}\right)$ to origin hub $k$ $\left(k \in H_{o}\right)$ represented by $R O_{i k}$. The second component is the unit hub-hub transportation cost, $\alpha T R_{k m}$, while third component is the unit cost of transportation through road from destination hub $m$ to warehouse $j\left(j \in W_{d}\right)$, represented by $R D_{m j}$.

The decision variables capture shipment quantity, route choice, hubs located and the hubs disrupted across origin and destination states. While the hub location and hub disruption decisions form the structural variables of the robust formulation, the shipment quantity and route choice form the control variables. The set of decision variables are defined as follows: 
$x_{i k m j}^{t s} \quad$ Quantity of food grains flowing from origin warehouse $i$ to destination warehouse $j$ through intermodal hubs $k$ and $m$ in time period $t$ and scenario $s$, where, $i \in W_{d}, j \in W_{d}$ $, k \in H_{o}, m \in H_{d}, t \in T, s \in S$

$y_{i k m j}^{t s}=1$, if there is flow from origin warehouse $i$ to destination warehouse $j$ through origin hub $k$ and destination hub $m$ in time period $t$ and scenario $s$, where, $i \in W_{o}, j \in W_{d}$, $k \in H_{o}, m \in H_{d}, t \in T, s \in S, 0$ otherwise.

$z_{k t} \quad=1$, if hub $k$ is open in time period $t, 0$ otherwise

$w_{m t} \quad=1$, if hub $m$ is open in time period $t, 0$ otherwise

$\gamma_{k t} \quad=1$, if hub $k$ is disrupted in time period $t, 0$ otherwise

$\delta_{m t} \quad=1$, if hub $m$ is disrupted in time period $t, 0$ otherwise

Minimize $T R R=\sum_{s \in S} \frac{p_{s} \xi_{s}}{\theta_{s}}$

$\xi_{s}=T C_{s}+\sum_{c \in \Omega} \pi_{c} G(c)-\theta_{s}$

$T C_{s}=T R C_{s}+H C+R C_{s}+E C_{s}$

$T R C_{s}=\sum_{t, i, k, m, j}\left(1-\gamma_{k t}\right)\left(1-\delta_{m t}\right) C T_{i k m j} x_{i k m j}^{t s}$

The objective function (Equation (2)) minimizes the total relative regret, $T R R$, where $p_{s}$ is the probability associated with occurrence of scenario $s$. In this model, TRR is a normalized measure of total regret, $\xi_{s}$ (Equation (3)) which accounts for solution robustness with respect to the near optimal cost, $\theta_{s}$ for a particular scenario $s \in S$. The second term in Equation (3) calculates the total induced penalty from the set of all control constraints, $\Omega . \pi_{c}$ and $G(c)$ denote the unit 
penalty cost and total violation for constraint type $c \in \Omega$. Constraint penalty increases the regret while ensuring that higher degree infeasibilities donot fit into the scope of acceptable solutions, thus accounting for model robustness. As different from the penalty approximation for constrained optimization, this penalty approach penalizes the violations resulting from uncertain parameters and decision variables of the stochastic problem. The total sustainable freight network cost is estimated using (Equation (4)) as an aggregate of following economic and ecological cost components: (1) Transportation cost, $T R C_{s}$ (2) Hub location cost, $H C_{s}$ (3) Rerouting cost, $R C_{s}$, and (4) Environmental cost, $E C_{s}$. The first term in the objective function, $T R C_{s}$ (Equation (5)), sums the transportation cost of individual shipments transported across all the routes passing through non-disrupted origin and destination hubs for each scenario $s \in S$. The multiplication $\left(1-\gamma_{k t}\right)\left(1-\delta_{m t}\right)$ ensures that the aggregated cost eliminates the cost of transporting disrupted shipments.

$$
\begin{aligned}
& H C=H C_{o}+H C_{d} \\
& H C_{o}=\sum_{k, t}\left[F_{k} z_{k t}\left(1-z_{k(t-1)}+\gamma_{k(t-1)}\right)+F_{k}^{\prime} z_{k(t-1)}\left(1-z_{k t}\right)\right] \\
& H C_{d}=\sum_{m, t}\left[F_{m} w_{m t}\left(1-w_{m(t-1)}+\delta_{m(t-1)}\right)+F_{m}^{\prime} w_{m(t-1)}\left(1-w_{m t}\right)\right]
\end{aligned}
$$

Equation (6) estimates the total hub location cost, where $H C_{o}$ represents total hub location costs at the origin, whereas $H C_{d}$ denotes total hub location cost at the destination states. $H C_{o}$ is calculated as per Equation (7) and $H C_{d}$ is measured according to Equation (8). $F_{k}$ and $F_{m}$ are the fixed costs of opening the hubs $k\left(k \in H_{o}\right)$ and $m\left(m \in H_{d}\right)$ respectively, whereas, $F_{k}^{\prime}$ and $F_{m}^{\prime}$ are the fixed costs of closing the hubs. The hub location cost, $H C_{o}$, in Equation (7) considers the 
summation of cost for opening and closing of hubs in the origin state. In this equation, the hub closure cost for a particular time period is accounted into total hub location cost if and only if the following conditions are satisfied. (1) The hub was opened in the immediate previous time period and (2) The hub is closed in the current time period. The expression $z_{k(t-1)}\left(1-z_{k t}\right)$ ensures that above conditions are satisfied under closure and becomes one, with $z_{k(t-1)}=1$ and $z_{k t}=0$, for all values of $k$ and $t$. If the hub $k$ remains open for continuous time periods $\left(z_{k(t-1)}=1\right.$ and $\left.z_{k t}=1\right)$, and hub $k$ was not disrupted in the previous time period $\left(\gamma_{k(t-1)}=0\right)$, then neither hub closure, nor hub opening costs is incurred. In the other case, if hub $k$ remains open for continuous time periods $\left(z_{k(t-1)}=1\right.$ and $\left.z_{k t}=1\right)$ and there was a disruption in the previous time period $\left(\gamma_{k(t-1)}=1\right)$, the model is designed to incorporate hub opening cost as it is required to recover from disruption although the hub was previously opened. A similar effort is exercised for estimating the hub location cost of the destination state using Equation (8). The hub location costs are independent of the supply scenarios unlike other components of the total cost which are evaluated after the actual realization of the supply.

$$
\begin{aligned}
& R C_{s}=R C_{o s}+R C_{d s}+R C_{o d s} \\
& R C_{o s}=\sum_{t, i, k, m, j} \gamma_{k t}\left(1-\delta_{m t}\right) C_{i e m j} x_{i k m j}^{t s} \\
& R C_{d s}=\sum_{t, i, k, m, j}\left(1-\gamma_{k t}\right) \delta_{m t} C_{i k f j} x_{i k m j}^{t s} \\
& R C_{o d s}=\sum_{t, i, k, m, j} \gamma_{k t} \delta_{m t} C_{i e f j} x_{i k m j}^{t s}
\end{aligned}
$$

The third term of the objective function, $R C_{s}$, is calculated based on three cases described as follows. In the first case, disruption occurs at only origin hub, whereas in the second case, 
disruption occurs only at the destination hub, and in the last case, disruption occurs at both origin and destination hubs. The rerouting cost pertaining to the abovementioned three cases is obtained from Equations (10), (11), and (12) respectively, where $C_{i e m j}, C_{i k j}$, and $C_{i e f j}$ represent the associated unit transit charges for routing the flow through the origin emergency hub, $e$ and, destination emergency hub, $f$, as appropriate. Inevitably, above three cases can be qualified as independent and mutually exclusive, and thus for a general case, the rerouting cost for each scenario $s \in S, R C_{s}$ is estimated by simple summation of the three costs, as represented in Equation (9).

$$
\begin{aligned}
E_{s}= & E_{\phi s}\left(1-\gamma_{k t}, 1-\delta_{m t}, k, m\right)+E_{\phi s}\left(\gamma_{k t}, \delta_{m t}, e, f\right)+E_{\psi s}\left(1-\gamma_{k t}, 1-\delta_{m t}, k, m\right)+E_{\psi s}\left(\gamma_{k t}, 1-\delta_{m t}, e, m\right) \\
& +E_{\psi s}\left(1-\gamma_{k t}, \delta_{m t}, k, f\right)+E_{\psi s}\left(\gamma_{k t}, \delta_{m t}, e, f\right)
\end{aligned}
$$

where, $E_{\phi s}\left(K, L, k^{\prime}, m^{\prime}\right)=\sum_{t}\left(\varepsilon_{\phi}+\varepsilon_{\phi}^{\prime}\right)\left(\sum_{i, k} K A_{i k^{\prime} \phi}\left\lceil\frac{\sum_{m, j} x_{i k m j}^{t s}}{V_{\phi}}\right\rceil+\sum_{m, j} L A_{m^{\prime} j \phi}\left\lceil\frac{\sum_{i, k} x_{i k m j}^{t s}}{V_{\phi}}\right\rceil\right)$

and, $\quad E_{\psi s}\left(K, L, k^{\prime}, m^{\prime}\right)=\sum_{t, k, m}\left(\varepsilon_{\psi}+\varepsilon_{\psi}^{\prime}\right) K L A_{k^{\prime} m \psi}\left\lceil\frac{\sum_{i, j} x_{i k m j}^{t s}}{V_{\psi}}\right\rceil$

$\forall(K, L) \in\{0,1\}, \forall k^{\prime} \in H_{o} \cup\{e\}, \forall m^{\prime} \in H_{d} \cup\{f\}$

In Equation (4), EC denotes the total environmental costs and is calculated for each scenario as $E C_{s}=C_{\tau} E_{s}$, where $E_{s}$ is the total emissions (Equation (13)) for scenario $s \in S . C_{\tau}$ denotes the price of carbon tax presented in rupees per tonne of $\mathrm{CO}_{2}$ released. $E_{\phi s}\left(K, L, k^{\prime}, m^{\prime}\right)$ (Equation (14)) and $E_{\psi s}\left(K, L, k^{\prime}, m^{\prime}\right)$ (Equation (15)) estimate the total $\mathrm{CO}_{2}$ emissions as a function of binary variables, $K$ and $L$, and intermodal hub indices, $k^{\prime}$ and $m^{\prime}$, for road and rail transport respectively 
for each scenario $s \in S$. The values for $K, L, k^{\prime}$ and $m$ ' are different for different cases of disruption that were discussed earlier and are appropriately chosen for each case. For example, substituting $K=1-\gamma_{k t}, L=1-\delta_{m t}, k^{\prime}=k$, and $m^{\prime}=m$, in Equation (14) corresponds to emissions estimation from a particular road portion of the transport where neither of the origin and destination hubs is disrupted. Whereas, the combination $K=\gamma_{k t}, L=\delta_{m t}, k^{\prime}=e$, and $m^{\prime}=f$ in the same equation, corresponds to the emissions estimation from the particular road portion of the transport where either one or both of origin and destination hubs are disrupted, and the disrupted consignments are routed through emergency hubs $e$ or/and $f$ as appropriate. In another case, substituting $K=1-\gamma_{k t}, L=1-\delta_{m t}, k^{\prime}=k$, and $m^{\prime}=m$, in Equation (15) corresponds to emissions estimation from the particular rail portion of the transport where neither of the origin and destination hubs is disrupted. Emissions from rail transport corresponding to disrupted consignments are evaluated using similar conventions provided in Equation (13) for single/multiple hub disruption cases. The first two terms of Equation (13) correspond to emissions from road transport, whereas the next four terms correspond to total emissions from rail transport. This procedure of estimating emissions is in accordance with estimation technique provided by Schipper et al. (2009). $\varepsilon_{\phi}$ and $\varepsilon_{\phi}^{\prime}$ in Equations (14) and (15) are full and empty load $\mathrm{CO}_{2}$ emissions $\left(\mathrm{gCO}_{2} /\right.$ vehicle-km) respectively for road transport, whereas for rail, $\varepsilon_{\psi}$ and $\varepsilon_{\psi}^{\prime}$ hold similar meanings. $V_{\phi}$ is the capacity of a single truck, whereas $V_{\psi}$ is the capacity of a single rake. The emission conversion factors used in Equations (14) and (15) are expressed in terms of total equivalent grams of $\mathrm{CO}_{2}$ per vehicle-kilometer travelled. Ramachandra and Shwetmala (2009) proposed emission factors for road transport. The emission factors for rail transport are adopted 
from Kishore (2012). This procedure of estimating emissions is in accordance with estimation technique provided by Schipper et al. (2009). Table 3 highlights the remaining list of parameters.

$$
\begin{aligned}
& x_{i k m j}^{t s} \leq M y_{i k m j}^{t s}, \forall i, \forall k, \forall m, \forall j, \forall t, \forall s \\
& \sum_{i, m, j} y_{i k m j}^{t s} \leq\left(|Z|\left|W_{o} \| H_{d}\right|\left|W_{d}\right|\right) z_{k t}, \forall k, \forall t, \forall s \\
& \sum_{i, k, j} y_{i k m j}^{t s} \leq\left(|Z|\left|W_{o}\right|\left|H_{o}\right|\left|W_{d}\right|\right) w_{m t}, \forall m, \forall t, \forall s \\
& \gamma_{k t} \leq z_{k t}, \forall k, \forall t
\end{aligned}
$$

Table 3. List of problem parameters

\begin{tabular}{ll}
\hline Notation & Description \\
\hline$D_{j t}$ & Demand at warehouse $j$ in time period $t, j \in W_{d}, t \in T$ \\
$I_{i t s}$ & Inventory at warehouse $i$ observed in time period $t$ and scenario $s, i \in W_{o}$ \\
& $, t \in T, s \in S$ \\
$P_{i t}^{s}$ & Procurement at warehouse $i$ observed in time period $t$ and scenario $s$, \\
& $i \in W_{o}, t \in T, s \in S$ \\
$U_{k}$ & Intermodal handling capacity of hub $k, k \in H_{o}$ \\
$U_{m}$ & Intermodal handling capacity of hub $m, m \in H_{d}$ \\
$n_{o}$ & No. of origin hubs permitted to be disrupted in any time period \\
$n_{d}$ & No. of destination hubs permitted to be disrupted in any time period \\
$\lambda_{i t}$ & No. of trucks available at warehouse $i$ in time period $t, i \in W_{o}, t \in T$ \\
$\mu_{k t}$ & No of rakes available at origin hub $k$ in time period $t, k \in H_{o}, t \in T$ \\
$\rho_{m t}$ & No. of trucks available at destination hub $m$ in time period $t, m \in H_{d}$, \\
& $t \in T$ \\
$A_{i k \phi}$ & Road distance from warehouse $i$ to intermodal hub $k, i \in W_{o}, k \in H_{o}$ \\
$A_{m j \phi}$ & Road distance from intermodal hub $m$ to warehouse $j, m \in H_{d}, j \in W_{d}$ \\
$A_{k m \psi}$ & Rail distance from intermodal hub $k$ to intermodal hub $m, k \in H_{o}$, \\
& $m \in H_{d}$
\end{tabular}

$\delta_{m t} \leq w_{m t}, \forall m, \forall t$ 
The problem is subjected to constraints shown in the Equations (16) - (43). Equation (16) associates the shipment quantity and route selection variables through a large number, $M$ in such a way that there is positive movement only if there exists a route in the same direction. However, as part of the random search strategy, the value of decision variable $x_{i k m j}^{t s}$ is allowed to vary in between a finite range depending upon the size of the problem in which case, the value of $M$ can be decreased till the upper bound for $x_{i k m j}^{t s}$. Equations (17) and (18) make sure that flow of food grain is routed through a hub only when it is open. Similarly, Equations (19) and (20) establish the linking constraints between disruption and hub location variables.

$$
\begin{aligned}
& y_{i k m j}^{t s}=\left[\frac{x_{i k m j}^{t s}}{1+x_{i k m j}^{t s}}\right], \forall i, \forall k, \forall m, \forall j, \forall t, \forall s \\
& \sum_{k} z_{k t}=a, \forall t \\
& \sum_{m} w_{m t}=b, \forall t \\
& \sum_{i, k, m} x_{i k m j}^{t s} \geq D_{j t}, \forall j, \forall t, \forall s \\
& \sum_{k, m, j} x_{i k m j}^{t s} \leq I_{i t s}, \forall i, \forall t, \forall s \\
& I_{i(t-1) s}+P_{i t s}-\sum_{k, m, j} x_{i k m j}^{t s}=I_{i t s}, \forall i, \forall t, \forall s \\
& \sum_{i, m, j} x_{i k m j}^{t s} \leq U_{k} z_{k t}, \forall k, \forall t, \forall s \\
& \sum_{i, k, j} x_{i k m j}^{t s} \leq U_{m} w_{m t}, \forall m, \forall t, \forall s
\end{aligned}
$$

The function in Equation (21) avoids empty transport between origin and destination warehouses. Equations (22) and (23), restrict the number of hubs located in origin and destination hubs in any time period to $a$ and $b$ respectively. Furthermore, the demand, available inventory and flow 
balance constraints are denoted by Equations (24), (25) and (26) respectively. Equations (27) and (28) are used to represent intermodal hub capacity restrictions.

$$
\begin{aligned}
& \sum_{k, m, j}\left(1-\omega_{i}\right) x_{i k m j}^{t s} \leq \lambda_{i t} V_{\phi}, \forall i, \forall t, \forall s \\
& \sum_{i, m, j} x_{i k m j}^{t s} \leq \mu_{k t} z_{k t} V_{\psi}, \forall k, \forall t, \forall s \\
& \sum_{i, k, j} x_{i k m j}^{t s} \leq \rho_{m t} w_{m t} V_{\phi}, \forall m, \forall t, \forall s
\end{aligned}
$$

It is made sure that the food grain flow are restricted by vehicle capacity from Equation (29), (30) and (31). The binary parameter, $\omega_{i}$ is defined to distinguish between a hub node and a non-hub node in Equation (29), where $\omega_{i}$ equals 1 if $i^{\text {th }}$ origin warehouse is a potential hub and equals 0 otherwise. Furthermore, the equation signifies vehicle capacity restriction only at the origin nonhub nodes, whereas the Equations (30) and (31) are formulated to satisfy vehicle capacity restrictions at origin and destination hubs respectively.

$$
\begin{aligned}
& y_{i e m j}^{t s} \geq z_{k t}+\gamma_{k t}+w_{m t}-2, \forall i, \forall k, \forall m, \forall j, \forall t, \forall s, m \neq f \\
& y_{i k j j}^{t s} \geq w_{m t}+\delta_{m t}-1, \forall i, \forall k, \forall m, \forall j, \forall t, \forall s, k \neq e \\
& y_{i e f j}^{t s} \geq z_{k t}+w_{m t}+\gamma_{k t}+\delta_{m t}-3, \forall i, \forall k, \forall m, \forall j, \forall t, \forall s
\end{aligned}
$$

When a hub is disrupted, one possibility is that the flow may be routed through non-disrupted hubs. Since the non-disrupted hubs possess limited intermodal handling and vehicle capacities, there is larger possibility of traffic congestion and further flow disruption. Therefore, this paper, considers that demand of food grains is routed through the emergency hubs after disruption. The emergency hubs are associated with high intermodal capacity and higher transportation cost. In this regard, Equations (32), (33) and (34) make sure that emergency route is selected respectively for the three cases of disruption as described earlier.

$$
z_{e t}=1, \forall t \in T
$$




$$
\begin{aligned}
& w_{f t}=1, \forall t \in T \\
& \gamma_{e t}=0, \forall t \in T \\
& \delta_{f t}=0, \forall t \in T \\
& \sum_{k} \gamma_{k t}=n_{o}, \forall t \in T \\
& \sum_{m} \delta_{m t}=n_{d}, \forall t \in T
\end{aligned}
$$

Equations (35) and (36) ensure that emergency hubs are always open. Equations (37) and (38) ensure emergency hubs are not disrupted. The number of disrupted hubs in the origin and destination hubs are restricted to $n_{o}$ and $n_{d}$ respectively by Equations (39) and (40).

$$
\begin{aligned}
& x_{i k m j}^{t s}=0, \forall i, \forall j, \forall t, \forall s,(i, k) \in H_{o} \\
& x_{i k m j}^{t s}=0, \forall i, \forall j, \forall t, \forall s,(j, m) \in H_{d} \\
& x_{i k m j}^{t s} \geq 0, y_{i k m j}^{t s}, z_{k t}, \gamma_{k t}, w_{m t}, \delta_{m t} \in\{0,1\}, \forall i, \forall j, \forall k, \forall m, \forall t, \forall s
\end{aligned}
$$

The Equations (41) and (42) are formulated to ensure no flow between two hubs of a single state. Finally, Equation (43) are non-negativity and integrality constraints for the given problem.

The procurement quantity, $P_{i t s}$ of warehouse $i \in W_{o}$ in time period $t \in T$ and scenario $s \in S$ is considered as the source of supply uncertainty. The constraints directly interacting with the control variables that may generate positive violation due to the variation in values of stochastic elements of the formulation are called control constraints. In this problem, constraints represented by Equations (16) - (18), (21), (24) - (34), are the control constraints. The violations generated from these constraints are penalized as shown in Equation (3). The following aspects highlight the uniqueness of the formulation. The simultaneous minimization of transportation, hub location, rerouting and environmental costs in the presence of intentional disruption and supply uncertainty is unique to transportation modelling in food grain context. The vehicle capacity constraint in 
combination with demand and flow constraints associated with various types of linking constraints are dealt with the scenarios of uncertain supply.

The dataset for the aforementioned problem formulation are described in Tables 4,5 and 6 respectively. In Table $4, n_{1}, n_{2}, n_{3}, n_{4}, n_{5}$, and $n_{6}$ symbolize the number of origin warehouses, origin hubs, destination hubs, destination warehouse, time periods and scenarios respectively. For example, the set of time periods is defined as $T=\{1,2\}$ for small dataset, and $T=\{1,2,3\}$ for medium and large data sets. Similarly, the number of elements in each set is purely dependent on the problem instance defined in Table 4. Therefore, Tables 4, 5 and 6 provide the dataset range for all the sets and different problem parameters defined in this section.

Table 4. Problem set description

\begin{tabular}{lllllll}
\hline $\begin{array}{l}\text { Problem } \\
\text { set }\end{array}$ & $\begin{array}{l}\text { Origin } \\
\text { regions }\end{array}$ & $\begin{array}{l}\text { Destination } \\
\text { regions }\end{array}$ & $\begin{array}{l}\text { Configuration } \\
\left(n_{1}, n_{2}, n_{3}, n_{4}, n_{5}, n_{6}\right)\end{array}$ & $\begin{array}{l}\text { Number of } \\
\text { variables }\end{array}$ & $\begin{array}{l}\text { Number of } \\
\text { control } \\
\text { constraints }\end{array}$ & $\begin{array}{l}\text { Number of total } \\
\text { constraints }\end{array}$ \\
\hline Small & 3 & 3 & $(5,3,3,5,2,3)$ & 2724 & 9858 & 9976 \\
Medium & 3 & 4 & $(6,3,5,9,3,3)$ & 14628 & 53271 & 53493 \\
Large & 3 & 4 & $(10,4,4,10,3,3)$ & 28848 & 102816 & 103074 \\
\hline
\end{tabular}

Table 5. Region wise warehouse distribution

\begin{tabular}{|c|c|c|c|c|c|c|c|c|c|c|c|c|}
\hline \multirow{3}{*}{$\begin{array}{l}\text { Problem } \\
\text { set }\end{array}$} & \multicolumn{4}{|c|}{ Small } & \multicolumn{4}{|c|}{ Medium } & \multicolumn{4}{|c|}{ Large } \\
\hline & \multicolumn{2}{|c|}{ Origin } & \multicolumn{2}{|c|}{ Destination } & \multicolumn{2}{|c|}{ Origin } & \multicolumn{2}{|c|}{ Destination } & \multicolumn{2}{|c|}{ Origin } & \multicolumn{2}{|c|}{ Destination } \\
\hline & HW & NHW & HW & NHW & HW & NHW & HW & NHW & HW & NHW & HW & NHW \\
\hline Reg 1 & 1 & 1 & 1 & 0 & 1 & 1 & 1 & 0 & 1 & 2 & 1 & 0 \\
\hline Reg 2 & 1 & 1 & 1 & 1 & 1 & 2 & 1 & 2 & 2 & 2 & 1 & 2 \\
\hline Reg 3 & 1 & 0 & 1 & 1 & 1 & 0 & 2 & 1 & 1 & 2 & 2 & 1 \\
\hline $\operatorname{Reg} 4$ & - & - & - & - & - & - & 1 & 1 & - & - & 0 & 3 \\
\hline
\end{tabular}

*HW: hub warehouse, NHW: non hub warehouse, Reg 1: Region 1, Reg 2: Region 2, Reg 3: Region 3, Reg 4: Region 4 
Table 6. Numerical description of problem and algorithm parameters

\begin{tabular}{llll}
\hline Parameter & Value & Parameter & Value \\
\hline$F_{k}$ & Rs. $5 \times 10^{5}-$ Rs. $10 \times 10^{5}$ & $b$ & $3-5$ \\
$F_{m}$ & Rs. $8 \times 10^{5}-$ Rs. $12 \times 10^{5}$ & $n_{o}$ & 1 \\
$A_{i k \phi}$ & $75 \mathrm{~km}-1000 \mathrm{~km}$ & $n_{d}$ & 1 \\
$A_{k m \mu}$ & $100 \mathrm{~km}-1500 \mathrm{~km}$ & $U_{k}$ & $2 \times 10^{5} \mathrm{MT}-4 \times 10^{5} \mathrm{MT}$ \\
$A_{m j \phi}$ & $50 \mathrm{~km}-500 \mathrm{~km}$ & $U_{m}$ & $1.5 \times 10^{5} \mathrm{MT}-3 \times 10^{5} \mathrm{MT}$ \\
$\alpha$ & 0.8 & $C_{\phi}$ & $15 \mathrm{MT}$ \\
$D_{j t}$ & $2000-20000 \mathrm{MT}$ & $C_{\psi}$ & $4000 \mathrm{MT}$ \\
$I_{i 0}$ & $25000-100000 \mathrm{MT}$ & $\lambda_{i t}$ & $1000-5000$ \\
$|S|$ & 3 & $\mu_{k t}$ & $20-45$ \\
$P_{i t}^{1}$ & $400-7000 \mathrm{MT}$ & $\rho_{m t}$ & $1000-5000$ \\
$P_{i t}^{2}$ & $2000-35000 \mathrm{MT}$ & $N$ & 300 \\
$P_{i t}^{3}$ & $4000-70000 \mathrm{MT}$ & $E$ & 0.9 \\
$p_{1}$ & 0.2 & $\omega$ & 0.9 \\
$p_{2}$ & 0.3 & $c_{1}$ & 0.1 \\
$p_{3}$ & 0.5 & $c_{2}$ & 0.98 \\
$a$ & $3-4$ & & \\
\hline
\end{tabular}

\section{Solution methodology}

Intermodal supply network optimization problems that deal with evaluation of simultaneous shipment route and hub allocation decisions are often developed considering p-hub median problem (Ishfaq and Sox, 2011). According to Parvaresh et al. (2014) and Alumur and Kara, (2008), the problem in the presence of single period interdictions, also known as p-hub median problem with intentional disruption (PHMI) is NP-hard. Since, the mathematical model developed in the current paper is a further extension of PHMI formulation with uncertain supply, the present formulation can be considered atleast as hard as PHMI problem. While decomposing and linearizing the aforementioned formulation has high possibility of fetching inefficient results in 
real time for large datasets, evolutionary optimization is an effective surrogate for solving such complex problems. Parvaresh et al. (2014) employed simulated annealing and tabu search techniques to solve the PHMI problem. A genetic algorithm based approach with multiple start points was adopted by Azizi et al. (2016) to solve PHMI variants. Metaheuristics such as genetic algorithm (GA), chemical reaction optimization (CRO), simulated annealing (SA), and ant colony optimization (ACO) have been used to solve (Mogale et al. 2016; Asgari et al. 2013) food grain supply chain problems. However, these techniques lack the ability to fully explore and exploit the solution space. Therefore, in this paper, particle swarm optimization with differential evolution (PSODE) proposed by Epitropakis et al. (2012) which combines the benefits of PSO and DE is tailored and used to solve the proposed problem.

\subsection{Particle swarm optimization with differential evolution}

Particle swarm optimization (Eberhart and Kennedy 1995) and differential evolution (DE) algorithms have been used to address a number of complex optimization problems. Having said so, the individual capabilities of both the algorithms are insufficient to tackle immature convergence. Swarm intelligence based meta-heuristics possess the capability to exploit the solution space more than the efficiency to explore (Liu et al. 2010). Specifically, the exploitation capability of particle swarm optimization (PSO) is much better compared to several other algorithms (Onut et al. 2008). On the other hand, differential evolution (DE) algorithm has relatively higher ability to explore the solution space but lack the efficiency to exploit a given scope of solution (Liu et al. 2010). Therefore, given the individual abilities and relative benefits of PSO and DE algorithms with respect to other approaches in exploration and exploitation, combining the two approaches would significantly strengthen the directional efficiency of the search process in both dimensions (Epitropakis et al. 2012). A number of authors presented the 
scope of hybridizing perspectives to gain synergistic benefits from the two algorithms (Xin et al. 2011; Liu et al. 2010; Thangaraj et al. 2011). An integrated form of canonical PSO and DE algorithms was originally proposed by Epitropakis et al. (2012). PSODE is characterized by an efficient combination of swarm and evolutionary meta-heuristic approaches, capable of reaping faster convergence rates. This paper aims to appropriately tailor and implement this hybrid approach to tackle the proposed constrained non-linear discrete optimization problem.

The hybrid algorithm is initiated with two dissimilar populations of equal size, pop $_{1}$ and pop $_{2}$. The particle best from pop $_{1}$ is stored in pop $_{2}$. The members of pop $_{1}$ are arranged in descending order as per constraint violations and pop $_{2}$ members are linked according to their particle best values. The populations continue to evolve separately, while $50 \%$ population of pop $_{1}$ are updated according to PSO. The individuals that are found to be infeasible are subjected to the reflection operator according to Equation (44). The notations $c, g$, and $l$ represent the particle, position, and the iteration number of the variable $x_{c g}^{l}$ respectively. The index $c$ represents a member from the set of particles, $P \in\{1,2,3, \ldots, N\}$, where $N$ is the population size. The index $g$ represents a member from the set of decision variables, $Z \in\{1,2,3, \ldots, D\}$, where $D$ is the dimension of each particle (total number of decision variables). The index $l$ represents a member from the set of iterations, $I \in\{1,2,3, \ldots, E\}$, where $E$ is the maximum iteration size. It is important to note that the variable $x_{c g}^{l}$ may correspond to any type of decision variable shown in Figure 4. $x_{c g}^{\prime l}= \begin{cases}0.5\left(l b(g)+x_{c g}^{l}\right), & \text { if } x_{c g}^{l}<l b(g) \\ 0.5\left(u b(g)+x_{c g}^{l}\right), & \text { if } x_{c g}^{l}>u b(g) \\ x_{c g}^{l}, \quad \text { otherwise } & \end{cases}$ 
Next, DE procedure is deployed on pop $_{2}$. The mutation strategies used for each member of pop $_{2}$ are as shown in Equations (45), (46) and (47).

$x_{c g}^{l l}=x_{r_{1} g}^{l}+\chi\left(x_{r_{2} g}^{l}-x_{r_{3} g}^{l}\right)$

$x_{c g}^{l l}=x_{c g}^{l}+\chi\left(x_{(b e s t) g}^{l}-x_{c g}^{l}\right)+\chi\left(x_{r_{1} g}^{l}-x_{r_{2} g}^{l}\right)$

$x_{c g}^{l}=x_{r_{1} g}^{l}+\chi\left(x_{r_{2} g}^{l}-x_{r_{3} g}^{l}\right)+\chi\left(x_{r_{4} g}^{l}-x_{r_{5} g}^{l}\right)$

$r_{1}, r_{2}, r_{3}, r_{4}, r_{5}$ are picked from uniform distribution in between [1, D], $x_{(b e s t) g}^{l}$ is the best solution obtained for $t$ iterations. $\mathrm{D}$ is the dimension (total number of decision variables) of the particle. $\chi$ is the amplification factor with range [l $\left.\begin{array}{ll}0 & 2\end{array}\right]$. These strategies were chosen to ensure maximum diversity in the resulting solutions. The boundary conditions are satisfied with the help of Equation (48), where $w_{c g}^{l}$ represents violated offsprings and $w_{c g}^{l l}$ represents corrected offsprings. Subsequently, the procedure of selection according to DE is implemented on the offspring to restore the particle best members of pop $_{2}$ at the end of iteration $t$ according to Equation (49), where the function $G(X)$ estimates the violation of constraint.

$$
\begin{aligned}
& w_{c g}^{l}= \begin{cases}\left.2 l b(g)-w_{c g}^{l}\right), & \text { if } w_{c g}^{l}<l b(g) \\
\left.2 u b(g)-w_{c g}^{l}\right), & \text { if } w_{c g}^{l}>u b(g) \\
w_{c g}^{l}, \quad \text { otherwise }\end{cases} \\
& \text { pbest }_{c}^{l+1}= \begin{cases}W_{c}^{l}, & \text { if } f\left(W_{c}^{l}\right)<f\left(\text { pbest }_{c}^{l}\right) \cap G\left(W_{c}^{l}\right) \leq G\left(\text { pbest }_{c}^{l}\right) \\
\text { pbest }_{c}^{l}, & \text { otherwise }\end{cases}
\end{aligned}
$$

The key advantages of the above approach can be measured in terms of less time taken by the algorithm to arrive at near optimal solutions as compared to other hybrid versions and its potential to generate adequate solution diversity.

\subsection{Implementation of PSODE}


The implementation of PSODE to solve the proposed problem is described in this section.

\subsubsection{Particle encoding scheme}

The particle encoding scheme defines the way in which the decision variables of the problem are collectively arranged. The order of decision variables in this problem are arranged as, $x_{i k m j}^{t s}, y_{i k m j}^{t s}$, $z_{k t}, w_{m t}, \gamma_{k t}$, and $\delta_{m t}$. As described in section 4, a specific configuration of the problem is characterized by the value of $n_{1}, n_{2}, n_{3}, n_{4}, n_{5}$, and $n_{6}$ which symbolize the number of origin warehouses, origin hubs, destination hubs, destination warehouse, time periods and scenarios respectively. For an instance, where $n_{1}=6, n_{2}=3, n_{3}=5, n_{4}=9, n_{5}=3$, and $n_{6}=3$, the total number of variables amounts to 14628 . The dimension of the particle matrix is $[N, D]$, where, $N$ represents population size and $D$ represents total number of decision variables. A sample encoding scheme is shown in Figure 4.

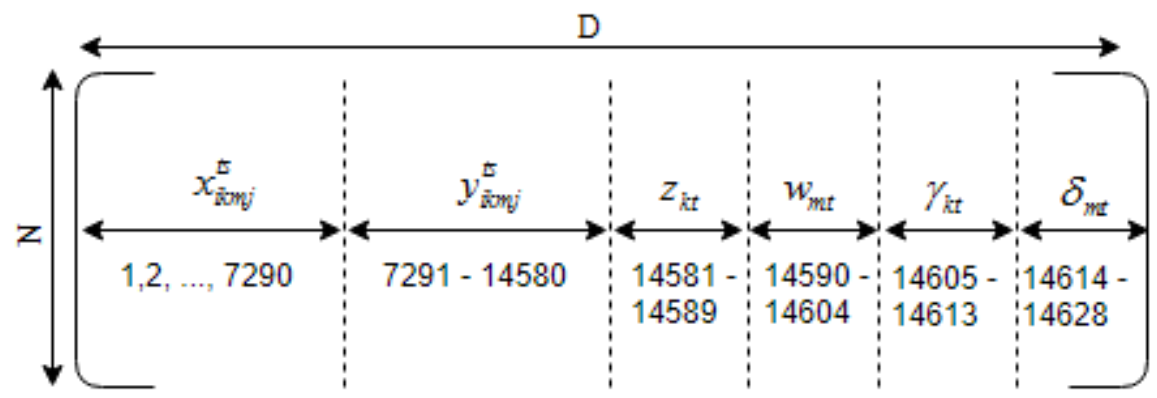

Figure 4. Particle encoding scheme for problem instance $(6,3,5,9,3,3)$

\subsubsection{Discretization}

In the course of particle evolution, PSO and DE often may cause the integer variables to get directed towards the outer region of the feasible space or may get transformed to continuous values. Discretization helps to forcefully restore integrality constraints on the corresponding violated variables. Integer variable discretization or binary variable discretization are used depending on the type of integer variables. The binary variables $y_{i k m j}^{t s}, z_{k t}, w_{m t}, \gamma_{k t}$, and $\delta_{m t}$ are treated with 
binary variable discretization scheme before calculation of fitness whereas the integer variables such as number of trucks or number of rains are subjected to integer variable discretization. Following this process, the solution string is passed on to the fitness function.

\subsubsection{Pattern generation}

The coding and decoding of a given particle is done through appropriate pattern generation. The patterns are generated for each expression of the objective function and constraints. In simple words, the patterns reveal the order in which the variables are accessed from the particle array. For example a pattern of a certain expression which reads $1,4,7,10$ and so on directs the access to $1^{\text {st }}$ , $4^{\text {th }}, 7^{\text {th }}$, and $10^{\text {th }}$ variables in the particle array. This simplifies the data structure for storing the variables and contributes to significant time savings for dealing with large problem scales and creates favorable coding environment for sensitivity analysis.

Table 7. Sample decision variable pattern sequences for demand constraint (Equation (24))

\begin{tabular}{lllll}
\hline $\begin{array}{l}\text { Right hand } \\
\text { side } \\
\left(D_{j t}\right)\end{array}$ & $j$ & $t$ & $s$ & Pattern for $x_{i k m j}^{t s}$ \\
\hline$D_{11}$ & 1 & 1 & 1 & $1,10,19, \ldots, 802$, \\
$D_{21}$ & 2 & 1 & 1 & $2,11,20, \ldots, 803$, \\
$D_{31}$ & 3 & 1 & 1 & $3,12,21, \ldots, 804$, \\
$D_{91}$ & 9 & 1 & 1 & $9,18,27, \ldots, 810$, \\
$D_{12}$ & 1 & 2 & 1 & $811,820,829, \ldots, 1612$, \\
$D_{93}$ & 9 & 3 & 1 & $1629,1638,1647, \ldots, 2430$ \\
$D_{11}$ & 1 & 1 & 2 & $2431,2440,2449, \ldots, 3232$ \\
$D_{93}$ & 9 & 3 & 3 & $6489,6498,6507, \ldots, 7290$ \\
\hline
\end{tabular}

Consider this is familiarized with the notation $\phi_{i}$ to represent a particular member of the pattern where $i$ takes values from 1 to the maximum number of decision variables. The pattern for $x_{i k m j}^{t s}$ in the demand constraint (Equation (24)) for different combinations of $j, t$, and $s$ are as shown 
in Table 7 for medium problem instance. The value of demand is constant and is independent of the realization of the scenario $s$. This can be seen in the last two rows of Table 7 where the demand values for scenarios 2 and 3 remain same as in scenario 1 for corresponding value of $j$ and $t$. A similar procedure is followed to evaluate the objective function and constraint expressions throughout this work.

\subsubsection{Penalty enforcement}

In robust optimization, penalty enforcement plays a significant role to capture model robustness while ensuring feasibility of control constraints. The global fitness or total relative regret is calculated as the relative expected sum of total regret with respect to the best solution achievable under a particular scenario. The total regret is the sum of total cost and penalty, $\Delta(X)$, for a given solution vector $X . \Delta(X)$ is calculated as per Equation (50). The value, $V_{n}(X)$ is the degree of violation of $n^{\text {th }}$ control constraint where $\pi_{n}$ represents penalty for violating the constraint.

$$
\Delta(X)=\sum_{n} \pi_{n} V_{n}(X)
$$

Given that $H_{l}(X) \leq B_{l}$ and $H_{m}(X)=B_{m}$ are the set of inequality and equality constraints of the problem respectively, $V_{n}(X)$ is calculated for each of these cases differently as per Equation (51)

$$
V_{n}(X)= \begin{cases}\left\{H_{l}(X)-B_{l}\right\}^{+} & \text {if } n=l \\ \left|H_{m}(X)-B_{m}\right| & \text { if } n=m\end{cases}
$$

\subsubsection{Flow diagram and pseudo code}

The flow diagram for PSODE application is illustrated in Figure 5 and its pseudo code is provided later in Figure 6. 


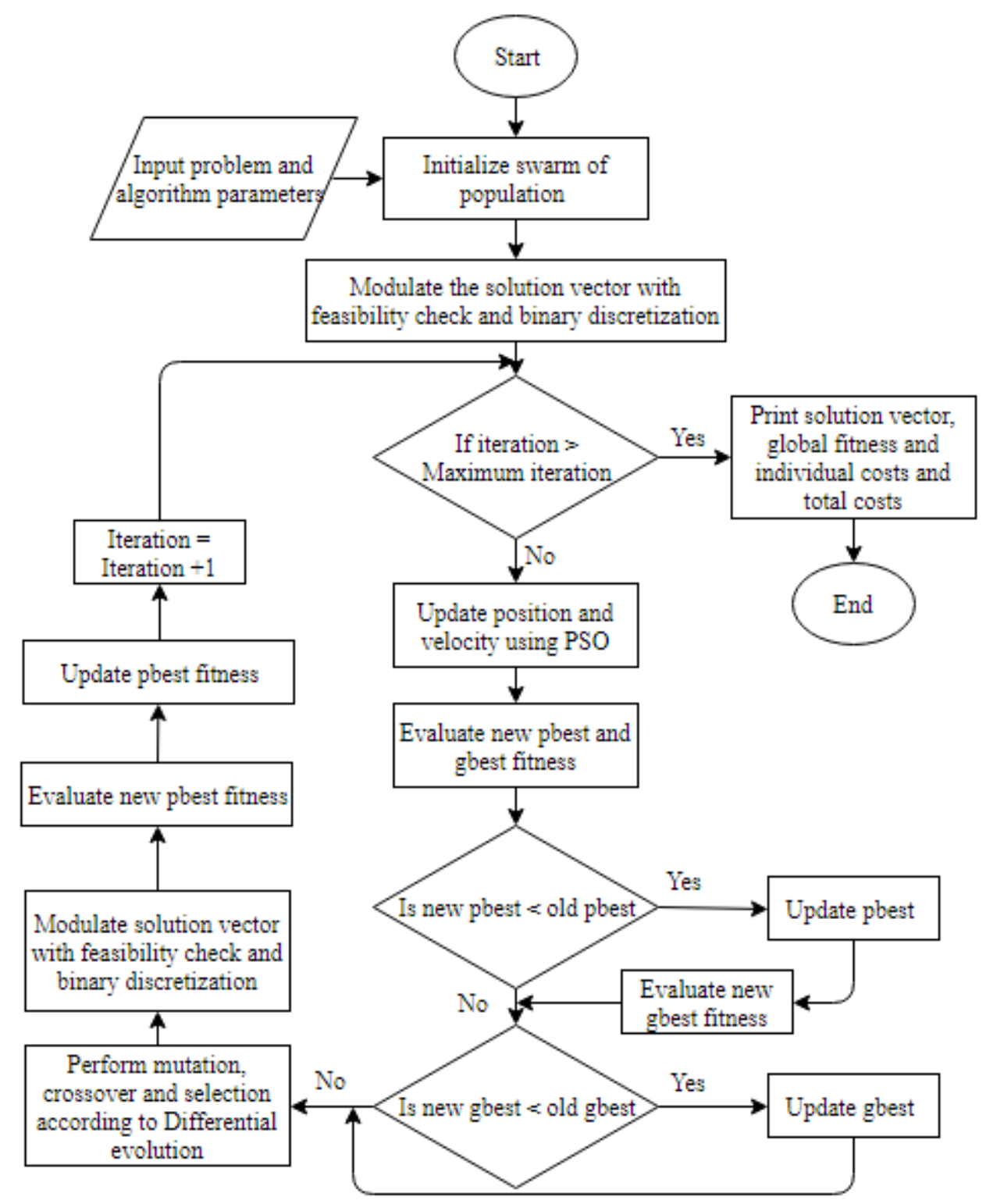

Figure 5. Flow diagram for PSODE implementation 


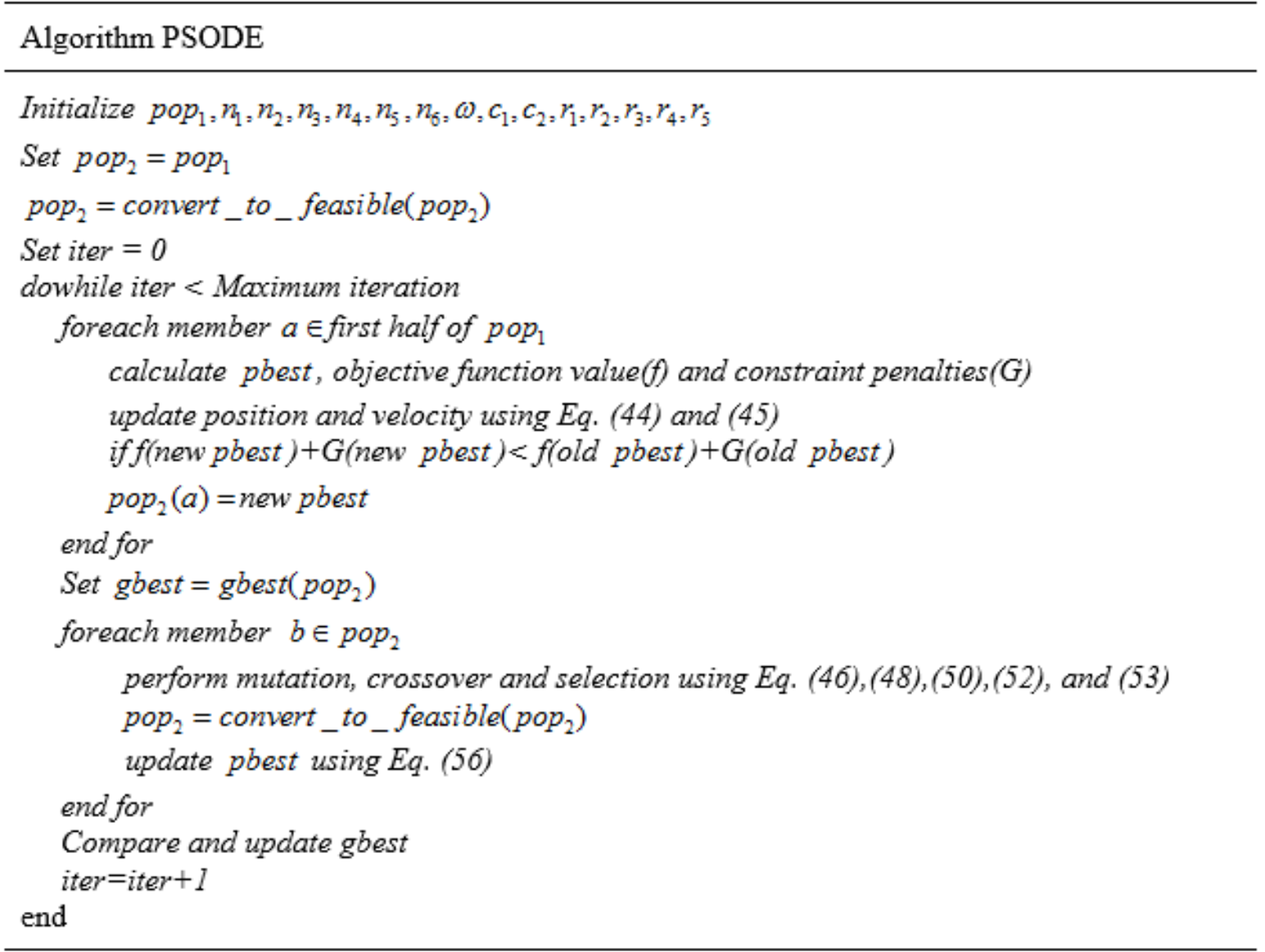

Figure 6. Pseudocode for PSODE

\section{Data and experiments}

In this research, the dataset for experiments is motivated from a diversified geographical scenario of South India. The type of food grain contemplated for the experimental study is rice. Simulation is conducted for food grain transport between two territories Tamil Nadu and Andhra Pradesh considered as origin and destination states, respectively. The demand for rice in Tamil Nadu is satisfied from the surplus stock available at Andhra Pradesh for multiple periods. The model is scrutinized on three samples of data with gradually augmented problem sizes (increasing number of variables). The data was assembled through field visit and by referring to different online sources (http://fci.gov.in,http://pdsportal.nic.in/main.aspx, https://www.fois.indianrail.gov.in/ 
foisweb/view/qry/TQFrgtCalcIN.jsp,). In general the articulation of total number of variables $\left(\Omega_{1}\right)$ and constraints $\left(\Omega_{2}\right)$ for the problem instance is illustrated in Equations (52) and (53). For every instance, the problem has to tackle with $O\left(n_{1} n_{2} n_{3} n_{4} n_{5} n_{6}\right)$ variables and constraints respectively.

$$
\begin{aligned}
& \Omega_{1}=2 n_{5}\left(n_{1} n_{2} n_{3} n_{4} n_{6}+n_{2}+n_{3}\right) \\
& \Omega_{2}=n_{5}\left[\begin{array}{l}
n_{6}\left\{\begin{array}{l}
5 n_{1} n_{2} n_{3} n_{4}+4\left(n_{1}+n_{2}+n_{3}\right)+n_{4}+n_{3} n_{4}\left(n_{2}-1\right)\left(n_{1}+n_{2}\right)+ \\
n_{1} n_{2}\left(n_{3}-1\right)\left(n_{3}+n_{4}\right)
\end{array}\right\}+3\left(n_{2}+n_{3}\right)+8
\end{array}\right]
\end{aligned}
$$

A wide range of decision variable compositions as shown in Table 4 are considered while augmenting the size of the problem. The smallest data set for the robust optimization model consists a total of 2724 continuous and discrete decision variables. The total number of constraints vary from 9976 in the small case to 103074 in the large case. Table 4 also shows the drastically increasing total number of control constraints with increasing problem size, thus, conforming to curse of dimensionality. Thus, the number of uncertainty scenarios chosen for the aforementioned problem is restricted to three as shown in Table 6 . As introduced earlier in section 4, scenarios 1 to 3 represent three levels procurement from low to high. Table 6 also illustrates the numerical range of procurement levels and probabilities associated with each scenario along with numerical values adopted for different parameters of problem and the algorithm. The region-wise distribution of warehouses for small, medium and large size instances are described in Table 5.

\section{Result and discussion}

The experiments were executed in MATLAB and performed on Windows 8, 64-bit Operating System comprising of $8 \mathrm{~GB}$ RAM and Intel Core i7 $2.2 \mathrm{GHz}$ processor. Section 7.1 presents results of solving the model for a case of deterministic supply while considering independent realizations of different procurement scenarios presented in Table 6. Section 7.2 presents the results of the 
model solved for simultaneous multiple realizations of procurement scenarios or in other words for the case of stochastic supply using robust optimization approach. The result were evaluated using PSODE algorithm initially which were later again evaluated and compared with particle swarm optimization (PSO) and firefly algorithms (FFA) in both the cases. The implementation of firefly algorithm was carried out by referring to stepwise execution outlined by Zhou et al. (2015) and Bottani et al. (2018).

\subsection{Single scenario case (deterministic supply)}

As discussed earlier in section 6, the problem is solved for three different sizes of problem (small, medium and large). Single scenario case captures the best solution or near optimal solution obtained for all three cases using PSODE, PSO and FFA. The evaluation of different cost components for different problem sets and algorithms from scenarios 1-3 are shown in Table 8. The average execution times reported in Table 8 are calculated as the average of CPU time and total iteration time elapsed until convergence. In Table 8, rerouting cost 1 is the cost incurred for rerouting the shipments through origin emergency hub when origin hub is disrupted while all destination hubs remain functional. Similarly, rerouting $\operatorname{cost} 2$ is the cost incurred for rerouting the shipments through destination emergency hub when only destination hub is disrupted while all origin hubs remain functional. Rerouting cost 3 represents the rerouting cost incurred for rerouting the shipments through origin and destination emergency hubs for the case where hubs at both origin and destinations are disrupted. The graphs shown in Figure 7 display the convergence of global fitness under scenarios 1-3 for different problem sets when solved using PSODE, FFA and PSO algorithms. For single scenario case, the global fitness in each iteration is calculated as the summation of total costs and total penalty incurred for the given dataset. Table 8 also captures the 
Table 8. Summary of results for deterministic optimization model for small, medium and large size instances for scenarios 1-3 using PSODE, PSO and FFA

\begin{tabular}{|c|c|c|c|c|c|c|c|c|c|c|}
\hline \multirow[t]{2}{*}{ Method } & \multirow{2}{*}{$\begin{array}{l}\text { Cost } \\
\text { and } \\
\text { time }\end{array}$} & \multicolumn{3}{|c|}{ Scenario 1 (Low procurement) } & \multicolumn{3}{|c|}{ Scenario 2 (Medium procurement) } & \multicolumn{3}{|c|}{ Scenario 3 (High procurement) } \\
\hline & & Small & Medium & Large & Small & Medium & Large & Small & Medium & Large \\
\hline \multirow[t]{9}{*}{ PSODE } & $\mathrm{TRC}$ & 375778880 & 1486777398 & 2616345970 & 484462769 & 1075134040 & 2695544092 & 467256190 & 1963267466 & 2174426614 \\
\hline & OLC & 2800000 & 3300000 & 5800000 & 2800000 & 3300000 & 5800000 & 2800000 & 3300000 & 4800000 \\
\hline & DLC & 4000000 & 8500000 & 6800000 & 4000000 & 8100000 & 6800000 & 4000000 & 8900000 & 6800000 \\
\hline & $\mathrm{RC} 1$ & 224699741 & 957509060 & 544228269 & 106325591 & 1403404581 & 969641741 & 371306882 & 713484539 & 1046791034 \\
\hline & $\mathrm{RC} 2$ & 201587948 & 807863694 & 866436683 & 226127412 & 538721504 & 461846626 & 93689995 & 210277126 & 624166016 \\
\hline & RC 3 & 43898432 & 98340621 & 119269437 & 25343173 & 11070123 & 259237976 & 207444881 & 141198536 & 94253756 \\
\hline & $\mathrm{EC}$ & 217658 & 1631804 & 4891106 & 250372 & 1346221 & 5468702 & 248510 & 1409392 & 4403309 \\
\hline & $\mathrm{TC}$ & 852982659 & 3363922577 & 4163771465 & 849309317 & 3041076469 & 4404339137 & 1146746458 & 3041837059 & 3955640729 \\
\hline & AET & 148.78 & 306.24 & 1125.48 & 155.7 & 312.87 & 1131.77 & 151.12 & 303.92 & 1158.28 \\
\hline \multirow[t]{9}{*}{ PSO } & TRC & 616358278 & 3212644109 & 4380395445 & 530893372 & 2939619926 & 4128594960 & 696735737 & 2983113894 & 4628493741 \\
\hline & OLC & 2800000 & 4300000 & 5800000 & 3300000 & 4300000 & 5400000 & 2800000 & 3300000 & 5400000 \\
\hline & DLC & 4000000 & 9200000 & 7400000 & 4000000 & 11300000 & 6400000 & 4000000 & 11900000 & 6800000 \\
\hline & RC 1 & 450992425 & 1848301688 & 2372508747 & 312526616 & 1804927746 & 2391929092 & 429674764 & 1975892374 & 2511134380 \\
\hline & RC 2 & 125191552 & 174353925.9 & 494920737 & 283112920 & 297313706.3 & 223360417 & 227323735 & 71469998 & 514545053 \\
\hline & RC 3 & 255253337 & 94542761.78 & 147505340 & 10968444 & 1748016.276 & 590555189 & 46643777 & 4950858 & 150106779 \\
\hline & $\mathrm{EC}$ & 253667 & 2124863.04 & 6272603 & 257063 & 2184627.72 & 5899326 & 251451 & 2045399 & 6533252 \\
\hline & $\mathrm{TC}$ & 1454849259 & 5345467348 & 7414802873 & 1145058416 & 5061394022 & 7352138984 & 1407429465 & 5052672524 & 7823013205 \\
\hline & AET & 128.46 & 257.34 & 1019.93 & 139.37 & 265.88 & 1025.44 & 138.57 & 269.62 & 1015.73 \\
\hline \multirow[t]{9}{*}{ FFA } & TRC & 814007895 & 3850105880 & 5258833949 & 606560419 & 3778470766 & 5615095149 & 622198459 & 3805507142 & 5269523279 \\
\hline & OLC & 3300000 & 3300000 & 5400000 & 2800000 & 3300000 & 5400000 & 3300000 & 3300000 & 5400000 \\
\hline & DLC & 4000000 & 8900000 & 6800000 & 4000000 & 8900000 & 6800000 & 4000000 & 8900000 & 6800000 \\
\hline & RC 1 & 638064295 & 2797980469 & 3299918851 & 588289519 & 2718041592 & 3432032078 & 664148627 & 2800427504 & 3147468950 \\
\hline & RC 2 & 283540391 & 1109652667 & 476515226 & 267104112 & 984689467 & 477594169 & 337505083 & 1160498745 & 469946506 \\
\hline & RC 3 & 262521569 & 731690554 & 225981378 & 360201881 & 816932817 & 151801461 & 118723062 & 676325747 & 354919972 \\
\hline & $\mathrm{EC}$ & 394405 & 3545602 & 8243705 & 343309 & 3503084 & 8798922 & 349731 & 3590252 & 8211589 \\
\hline & $\mathrm{TC}$ & 2005828556 & 8505175171 & 9281693108 & 1829299239 & 8313837726 & 9697521780 & 1750224962 & 8458549391 & 9262270296 \\
\hline & AET & 134.91 & 255.46 & 1008.39 & 135.26 & 249.66 & 1024.89 & 140.69 & 264.84 & 1013.84 \\
\hline
\end{tabular}

*TRC: Transportation cost, OLC: Origin hub location cost, DLC: Destination hub location cost, RC1: Rerouting cost 1, RC2: Rerouting cost 2,

RC3: Rerouting cost 3, EC: Environmental cost, TC: Total cost , AET: Average executive time. All the cost in Rupees and time in second 
average execution times required for convergence of global fitness using PSODE, PSO and FFA for all instances.

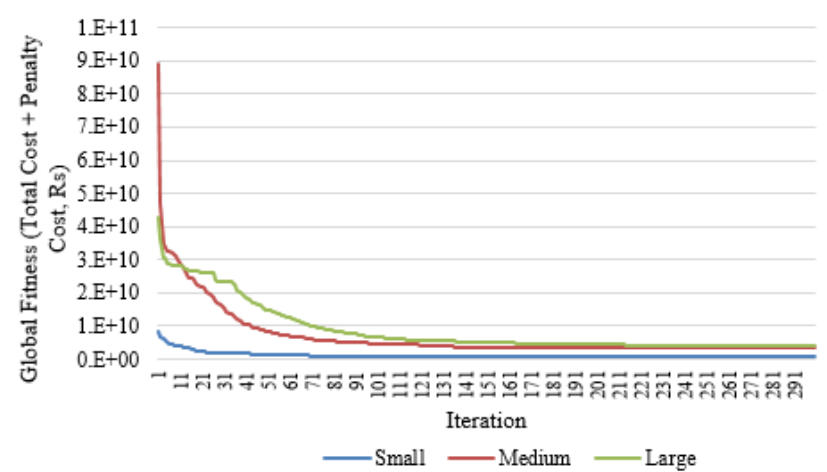

(a) Scenario 1 (Low procurement), PSODE

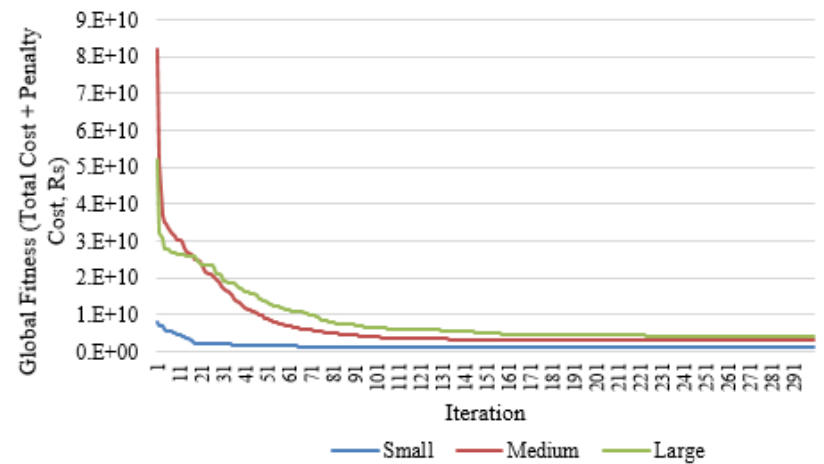

(c) Scenario 3 (High procurement), PSODE

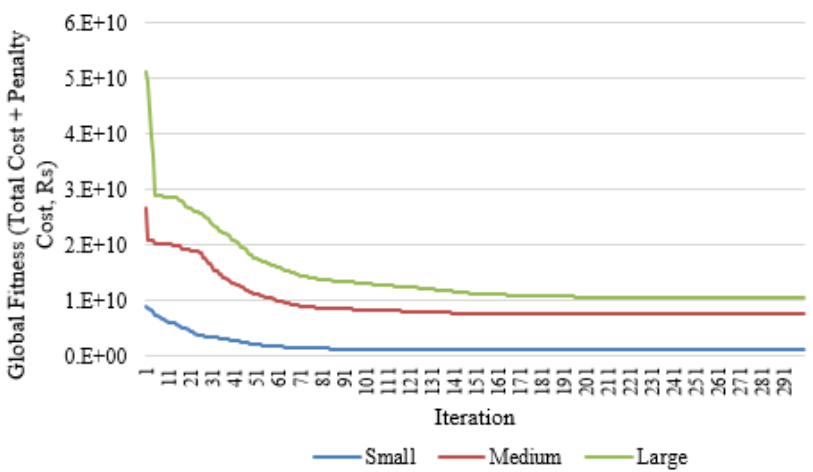

(e) Scenario 2 (Medium procurement), PSO

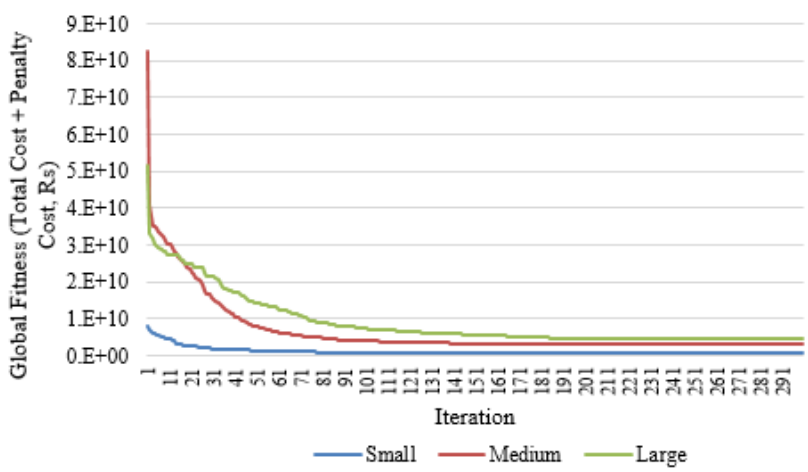

(b) Scenario 2 (Medium procurement), PSODE

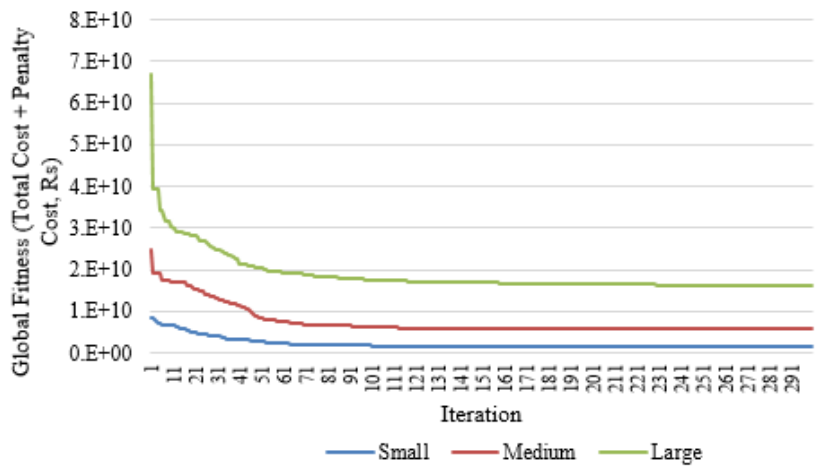

(d) Scenario 1 (Low procurement), PSO

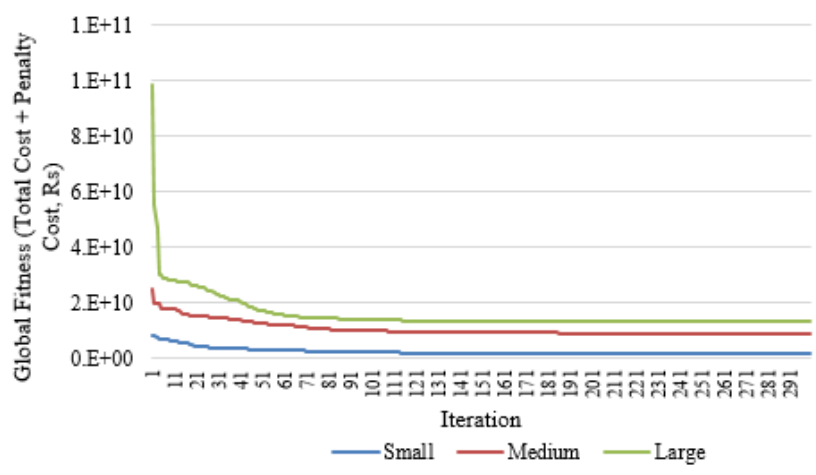

(f) Scenario 3 (High procurement), PSO

Figure 7. Convergence of global fitness by PSODE, PSO and FFA for different problem sizes and procurement scenarios under deterministic supply. 


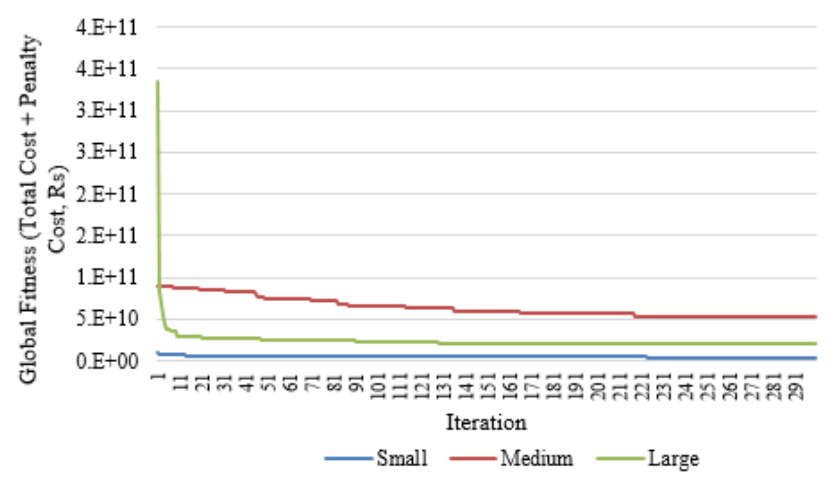

(g) Scenario 1 (Low procurement), FFA

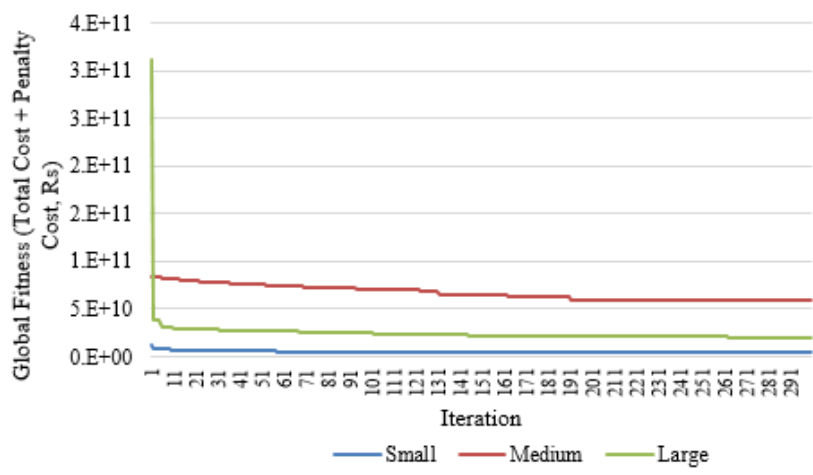

(h) Scenario 2 (Medium procurement), FFA

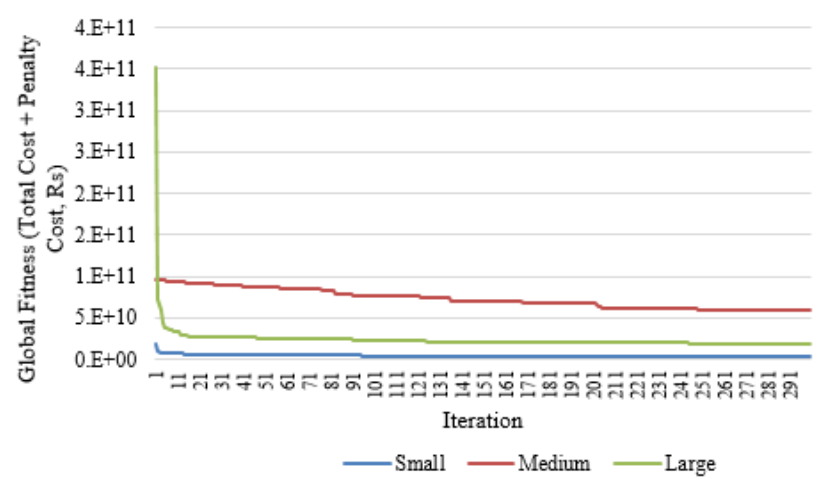

(i) Scenario 3 (High procurement), FFA

Figure 7. Continued.

\subsection{Multiple scenario case (stochastic supply)}

Analogous to the deterministic case, the computational results of the proposed model for stochastic case of supply (unknown procurement) are recorded for small, medium and large size problems as shown in Table 9. However, in this case, the results include expected costs associated with transportation, rerouting, environmental and total costs. The cost associated with structural variables (origin and destination facility location cost) remain constant for all scenarios. In Table 9, expected rerouting cost 1-3 correspond to rerouting costs obtained for respective scenarios of disruption as defined earlier in section 7.1. Table 9 also presents penalty incurred for not being able to fully satisfy the control constraints while dealing with the uncertain procurement. Later, regret associated with a particular procurement scenario $\left(\xi_{1}, \xi_{2}\right.$, and $\left.\xi_{3}\right)$ calculated using Equation 
(3) are reported. Total relative regret calculated using Equation (2) is reported in the last second row. The largest size of the model with 28848 variables and 103074 constraints was solved within $1.5 \mathrm{~h}$ of computational time. Figure 8 shows the convergence of global fitness or total relative regret for different sizes of the problem solved by all three algorithms. It is found that PSODE consumes slightly higher average execution than other traditional metaheuristics evident from Tables 8 and 9. This is attributed to the additional operators and penalty enforcement mechanisms embedded in PSODE as compared to the less number of operators in PSO and FFA. However, this is compensated by the huge difference in the solution quality (minimum cost) obtained in the case of PSODE as compared to the costs obtained by other two algorithms. The detailed discussion about the results obtained and comparative analysis in terms of solution quality, solution and model robustness between the algorithms are reported in section 7.3 for different datasets. 
Table 9. Summary of results for robust optimization model for small, medium and large size instances using PSODE, PSO and FFA

\begin{tabular}{|c|c|c|c|c|c|c|c|c|c|}
\hline \multirow{2}{*}{ Cost and time } & \multicolumn{3}{|l|}{ PSODE } & \multicolumn{3}{|l|}{ PSO } & \multicolumn{3}{|l|}{ FFA } \\
\hline & Small & Medium & Large & Small & Medium & Large & Small & Medium & Large \\
\hline ETRC & 585537042 & 2263235780 & 3612731129 & 809548896.7 & 3173750669 & 5525786615 & 586625450 & 3762641379 & 5600492594 \\
\hline OLC & 2800000 & 3300000 & 4800000 & 2800000 & 3300000 & 5800000 & 2800000 & 3300000 & 5800000 \\
\hline DLC & 4000000 & 8500000 & 6800000 & 4000000 & 8900000 & 8600000 & 4000000 & 8900000 & 6800000 \\
\hline ERC 1 & 467308304 & 1463208020 & 2161881058 & 750345260.6 & 2544885936 & 2971071011 & 581954495 & 2758882111 & 3195542251 \\
\hline ERC 2 & 352356300 & 1399734557 & 801119644 & 410152138 & 188158040 & 988973089 & 433699665 & 1036913034 & 1227736899 \\
\hline ERC 3 & 316763601 & 711716036 & 738505574 & 454360003.3 & 285718769 & 295324692 & 306246531 & 794983942 & 542200607 \\
\hline $\mathrm{EC}$ & 324313 & 2363397.43 & 6449488 & 436815.462 & 2397197 & 8375743 & 334231 & 3577746 & 9594260 \\
\hline ETC & 1729089561 & 5852057790 & 7332286893 & 2431643114 & 6207110611 & 9803931149 & 1915660372 & 8369198212 & 10588166611 \\
\hline PI & 1701710691 & 6480780667 & 8333583184 & 2824162640 & 6234048694 & 10507609228 & 1684215334 & 8345711892 & 10213078856 \\
\hline PI/ ETC & 0.98 & 1.11 & 1.14 & 1.16 & 1.00 & 1.07 & 0.88 & 1.00 & 0.96 \\
\hline$\xi_{1}$ & 915520090 & 3170375508 & 4323749399 & 1487651559 & 3550942924 & 103950214.4 & 190054621 & 16083560561 & 820246040 \\
\hline$\xi_{2}$ & 1082161331 & 2609213133 & 2573800657 & 1772393183 & 1790630029 & 4993385107 & 265362187 & 9323655701 & 650518186 \\
\hline$\xi_{3}$ & 578733292 & 2706053966 & 3418639924 & 863666935 & 222396436 & 1959969438 & 746236940 & 8553563461 & 2360428708 \\
\hline TRR & 0.85 & 0.88 & 0.81 & 0.90 & 0.20 & 0.22 & 0.16 & 0.18 & 0.08 \\
\hline AET & 646.78 & 2154.06 & 5247.3 & 594.96 & 1968.49 & 5032.94 & 611.13 & 2071.64 & 4184.14 \\
\hline
\end{tabular}

*ETRC: Expected Transportation cost, OLC: Origin hub location cost, DLC: Destination hub location cost, ERC1: Expected rerouting cost 1, ERC2: Expected rerouting cost 2,ERC3: Expected rerouting cost 3, EEC: Expected environmental cost, ETC1: Expected total cost 1, ETC2: Expected total cost 2, PI: Penalty Incurred, TRR: Total relative regret, AET: Average executive time. All the cost in Rupees and time in second 


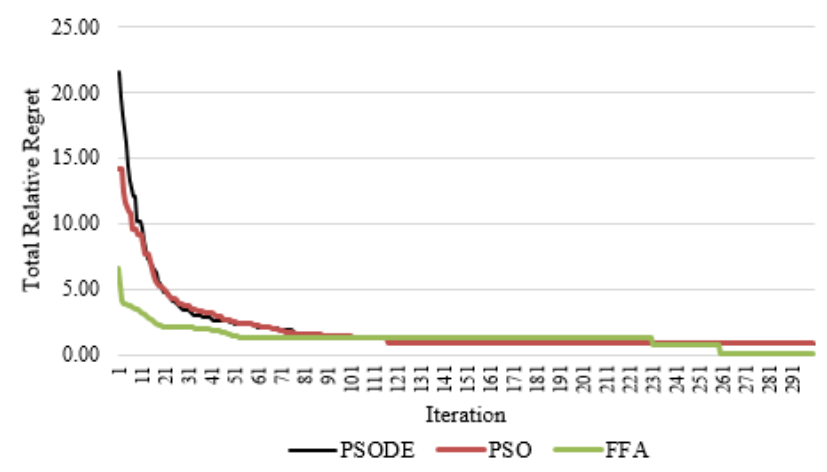

(a) Small

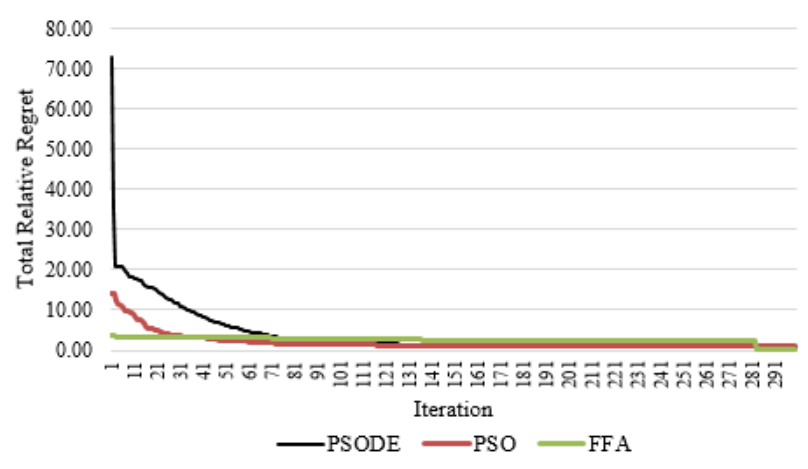

(b) Medium

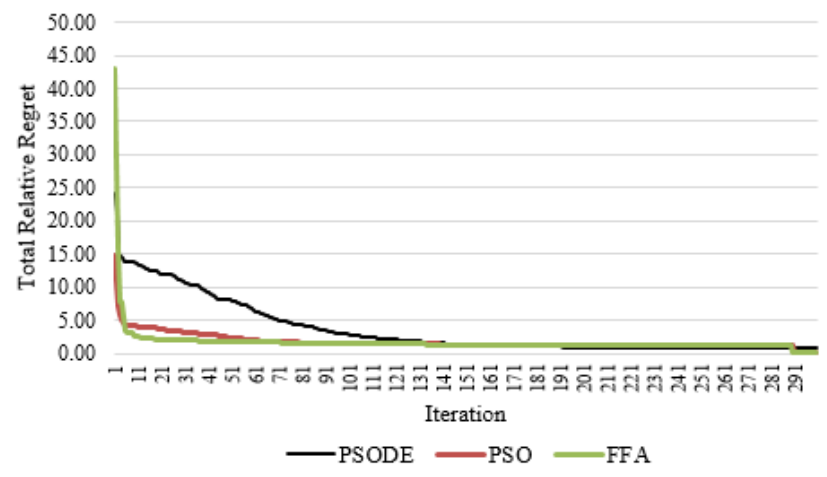

(c) Large

Figure 8. Convergence of global fitness (total relative regret) by PSODE, PSO and FFA for different problem sizes under uncertain supply

\subsection{Implication}

The theoretical implications of the current research are highlighted as follows. The study develops a unique mathematical model with focus to simultaneously capture uncertainty, intentional disruptions and environmental issues in the context of Indian food grain transportation. The decision variables of the model capture near optimal shipment quantity, hub location decisions and route selection while considering supply side uncertainty. The solution methodology combines the robust optimization approach and PSODE strategy to achieve near optimal solutions for the proposed problem. The solution robustness is evaluated in terms of total relative regret and the model robustness is captured by considering penalty for the control constraints. Specifically, 
absolute and relative penalty incurred (ratio of penalty incurred to total expected costs) are the two different types of measures (Table 9) incorporated to measure model robustness. Further, it is found that the proposed method is able to offer superior quality of solution with respect to two other state-of the art metaheuristic approaches.

Large supply chain organizations often face difficulty while making freight booking and facility location decisions and are generally taken based on historical data. Given the large scale network of food grain supply chains, the results of the proposed model are significantly conducive to key decision makers and stakeholders, especially while dealing with uncertainty in food grain supply and intentional disruptions (disruptions due to war and terrorists attacks). With respect to the present problem, the minimized total relative regret is found to be less than unity for all cases. It is important to note that although PSO and FFA algorithms are able to provide lesser values of total relative regret, the quality of solution (Total cost and Expected total cost) provided by PSODE is superior for all datasets (Tables 8 and 9). This guarantees the replicability of the proposed approach with respect to different problem sizes. The relative regret measures the relative deviation of the robust solution from the solution obtained in the case of individual scenarios. It does not take into consideration the inferiority of the solutions obtained as it is the case for PSO and FFA (Table 8) with respect to PSODE. It could be misleading to completely rely on the measure without considering the relative performance of the algorithms in terms of solution quality. Therefore, it is important to simultaneously consider model robustness while evaluating the overall robustness of the proposed approach. PSODE provides a higher value of model robustness compared to other approaches considered in this study depicted by its higher value of relative penalty measure shown in Table 9 for all datasets except for one case (small data set with PSO) which might be attributed to insignificant problem size. A higher value of relative penalty 
measure indicates low level of expected total cost and thus higher solution quality. Considering the given scale of the practical problem and its computationally intractable nature, and attractive average execution times, the proposed approach is found to be capable, flexible and replicable in the current context and could be tailored to suit wide range of problems subject to uncertain environment. The competition of the government organization with the private market depends on additional uncontrollable factors such as selling price to farmers (minimum support price), price of food grains offered by private parties, procurement patterns, and weather conditions to name a few. Since the focus of the current work is restricted to transportation, the advantages of the proposed approach cannot be directly estimated in terms of competitive advantage but can be indirectly estimated based on the ability of the robust and sustainable modelling approach to cause significant cost savings to the government organizations and country at large towards successful implementation of sustainable transportation.

\section{Conclusion and future scope}

This paper proposes a mixed integer non-linear discrete robust optimization model for food grain transportation problem in the presence of uncertain supply and intentional disruptions in Indian context. The proposed model fosters a holistic and sustainable perspective by considering transportation cost, intermodal facility location cost, rerouting costs and environmental cost while minimizing the total relative regret associated with total cost. The constraints of the model adhere to vehicle capacity, hub capacity, and emergency hub restrictions along with food grain demand and flow balance constraints. In the problem, shipment quantity and shipment route decisions were designed as the control variables while hub location and disruption variables as structural variables. A self-tailored hybrid metaheuristic, PSODE is employed to tackle the resulting 
complex problem in real time. The discrete and robust versions of the model was tested and compared for small, medium and large size problems using two other state of the art metaheuristics. Later the results of the robust model was verified against model and solution robustness for different levels of procurement scenarios. Results illustrate that the hybrid metaheuristic was able to solve the problem with attractive computational times and arrive at higher quality of solution for all the instances.

The limitations of the proposed research are highlighted as follows. Firstly, the current study focusses on considering discrete scenarios which may be further perfected by capturing the continuous distribution of uncertain parameters. Secondly, the proposed model is developed as single objective optimization approach whereas in practical it is important to capture the trade-off between the economic and environmental facets of sustainability. In this line, multi-objective treatment of the proposed model to excavate practically implementable tradeoffs between total shipment cost and carbon emissions is an open research question. Thirdly, the current study assumes carbon tax scheme for estimation of GHG emissions costs. However this may not be generalized to developed countries where carbon trading is being implemented. In such cases, the current study requires deeper analysis to investigate the relative benefits of resorting to an efficient emission conversion strategy. Nevertheless, this research opens new avenues for research in the arena of food grain transportation. Further, the scope of model implementation can be widened by designing similar supply network for other geographically wide spread territories with slight modification as per practical requirements, especially where food grains are the staple food. 


\section{References}

Accorsi, R., S. Cholette, R. Manzini, C. Pini, and S. Penazzi. 2016. "The land-network problem: ecosystem carbon balance in planning sustainable agro-food supply chains." Journal of Cleaner Production 112: 158-171.

Ahumada, O., J. R. Villalobos, and A. N. Mason. 2012. "Tactical planning of the production and distribution of fresh agricultural products under uncertainty." Agricultural Systems 112: $17-26$.

Akkerman, R., P. Farahani, and M. Grunow. 2010. "Quality, safety and sustainability in food distribution: a review of quantitative operations management approaches and challenges." Or Spectrum 32(4): 863-904.

Alumur, S. and B. Y. Kara. 2008. "Network hub location problems: The state of the art." European journal of operational research 190(1): 1-21.

Amin, S. H. and G. Zhang. 2013. "A multi-objective facility location model for closed-loop supply chain network under uncertain demand and return.” Applied Mathematical Modelling 37 (6): 4165-4176.

Asgari, N., R. Z. Farahani, H. Rashidi-Bajgan, and M. S. Sajadieh. 2013. "Developing model based software to optimise wheat storage and transportation: a real-world application." Applied Soft Computing 13(2): 1074-1084.

Azizi, N., S. Chauhan, S. Salhi, and N. Vidyarthi. 2016. The impact of hub failure in hub-andspoke networks: Mathematical formulations and solution techniques. Computers \& Operations Research 65:174-188. 
Barbosa-Póvoa, A.P., C. da Silva, and A. Carvalho. 2017. "Opportunities and challenges in sustainable supply chain: An operations research perspective." European Journal of Operational Research.

Ben-Tal, A., D. Den Hertog, and J. P. Vial. 2015. Deriving robust counterparts of nonlinear uncertain inequalities. Mathematical programming 149(1-2): 265-299.

Bertsimas, D. and M. Sim. 2004. "The price of robustness." Operations research 52(1):35-53.

Blackhurst, J., C. W. Craighead, D. Elkins, and R. B. Handfield. 2005. “An empirically derived agenda of critical research issues for managing supply-chain disruptions." International journal of production research 43 (19): 4067-4081.

Borodin, V., J. Bourtembourg, F. Hnaien, and N. Labadie. 2016. "Handling uncertainty in agricultural supply chain management: A state of the art." European Journal of Operational Research254 (2): 348-359.

Bortolini, M., M. Faccio, E. Ferrari, M. Gamberi, and F. Pilati. 2016. "Fresh food sustainable distribution: cost, delivery time and carbon footprint three-objective optimization." Journal of Food Engineering 174: 56-67.

Bottani, E., P. Centobelli, R. Cerchione, L. Gaudio, and T. Murino. 2017. "Solving machine loading problem of flexible manufacturing systems using a modified discrete firefly algorithm." International Journal of Industrial Engineering Computations 8(3): 363-372.

Bozorgi-Amiri, A., M.S. Jabalameli, M. Alinaghian, and M. Heydari. 2012. "A modified particle swarm optimization for disaster relief logistics under uncertain environment." The International Journal of Advanced Manufacturing Technology 60 (1-4): 357-371. 
Chopra, S and M. S. Sodhi. 2004. "Managing risk to avoid supply chain breakdown [J]." MIT Sloan Management Review 46: 53-61.

Diabat, A., K. Govindan, and V. V. Panicker. 2012. "Supply chain risk management and its mitigation in a food industry." International Journal of Production Research 50 (11): 3039-3050.

Eberhart, R. and J. Kennedy. 1995. A new optimizer using particle swarm theory. In Micro Machine and Human Science, 1995. MHS'95., Proceedings of the Sixth International Symposium on (pp. 39-43). IEEE.

Epitropakis, M. G., V. P. Plagianakos, and M.N. Vrahatis. 2012. "Evolving cognitive and social experience in particle swarm optimization through differential evolution: a hybrid approach.” Information Sciences 216: 50-92.

Garnett, T. 2011. "Where are the best opportunities for reducing greenhouse gas emissions in the food system (including the food chain)?.” Food policy 36: S23-S32.

Garrone, P., M. Melacini, and A. Perego. 2014. "Surplus food recovery and donation in Italy: the upstream process.” British Food Journal 116 (9):1460-1477.

Ge, H., R. Gray, and J. Nolan. 2015. "Agricultural supply chain optimization and complexity: A comparison of analytic vs simulated solutions and policies." International Journal of Production Economics159: 208-220.

Govindan, K., A. Jafarian, and V. Nourbakhsh. 2015. "Bi-objective integrating sustainable order allocation and sustainable supply chain network strategic design with stochastic demand 
using a novel robust hybrid multi-objective metaheuristic.” Computers \& Operations Research 62: 112-130.

Govindan, K., M. Fattahi, and E. Keyvanshokooh. 2017. "Supply chain network design under uncertainty: A comprehensive review and future research directions." European Journal of Operational Research 263 (1): 108-141.

Hachicha, N., B. Jarboui, and P. Siarry. 2011. “A fuzzy logic control using a differential evolution algorithm aimed at modelling the financial market dynamics." Information Sciences 181 (1): 79-91.

Hasany, R.M. and Y.Shafahi. 2017. "Two-stage stochastic programming for the railroad blocking problem with uncertain demand and supply resources." Computers \& Industrial Engineering 106: 275-286.

Huang, H., Y. He, and D. Li. 2018. "Pricing and inventory decisions in the food supply chain with production disruption and controllable deterioration." Journal of Cleaner Production, 180: 280-296.

Huang, S.H. and P.C. Lin. 2010. “A modified ant colony optimization algorithm for multi-item inventory routing problems with demand uncertainty." Transportation Research Part E: Logistics and Transportation Review 46 (5): 598-611.

INCCA, 2010. India: Greenhouse Gas Emissions 2007. Indian Network for Climate Change Assessment (INCCA), The Ministry of Environment \& Forests, Government of India, 63.

IPCC, 2014. Transportation. Fifth Assessment Report. 
Ishfaq, R. and C. R. Sox. 2011. "Hub location-allocation in intermodal logistic networks.” European Journal of Operational Research 210(2): 213-230.

Jalilvand-Nejad, A., R. Shafaei, and H. Shahriari. 2016. "Robust optimization under correlated polyhedral uncertainty set.” Computers \& Industrial Engineering 92:82-94.

Jung, J.Y., G. Blau, J.F. Pekny, G.V. Reklaitis, and D. Eversdyk. 2004. "A simulation based optimization approach to supply chain management under demand uncertainty." Computers \& chemical engineering 28 (10): 2087-2106.

Khatami, M., M. Mahootchi, and R.Z. Farahani. 2015. 'Benders' decomposition for concurrent redesign of forward and closed-loop supply chain network with demand and return uncertainties." Transportation Research Part E: Logistics and Transportation Review 79: $1-21$.

Kishore, E. A. 2012. “Carbon emissions from Indian Railways: An estimation for transportation of goods during 2010-2011.”

Laguna, M. 1998. “Applying robust optimization to capacity expansion of one location in telecommunications with demand uncertainty.” Management Science 44(11-part-2): S101S110.

Liu, H., Z. Cai, and Y. Wang. 2010. "Hybridizing particle swarm optimization with differential evolution for constrained numerical and engineering optimization." Applied Soft Computing 10(2): 629-640. 
Liu, Y., H. Lei, D. Zhang, and Z. Wu. 2018. "Robust optimization for relief logistics planning under uncertainties in demand and transportation time." Applied Mathematical Modelling 55: 262-280.

Lium, A. G., T. G. Crainic, and S. W. Wallace. 2009. "A study of demand stochasticity in service network design.” Transportation Science 43 (2): 144-157.

Mangla, S.K., S. Luthra, N. Rich, D. Kumar, N. P. Rana, and Y. K. Dwivedi. 2018. "Enablers to implement sustainable initiatives in agri-food supply chains." International Journal of Production Economics 203: 379-393.

Maiyar, L. M. and J. J. Thakkar. 2019. "Environmentally conscious logistics planning for food grain industry considering wastages employing multi objective hybrid particle swarm optimization." Transportation Research Part E: Logistics and Transportation Review (Accepted).

Maiyar, L. M., and J. J. Thakkar. In press. "Modelling and analysis of intermodal food grain transportation under hub disruption towards sustainability" International Journal of Production Economics.

Maiyar, L. M., J. J. Thakkar, A. Awasthi, and M. K. Tiwari. 2015. "Development of an effective cost minimization model for food grain shipments." IFAC-PapersOnLine 48 (3): 881-886.

Mogale, D. G., S. K. Kumar, and M. K. Tiwari. 2016. "Two stage Indian food grain supply chain network transportation-allocation model.” IFAC-PapersOnLine 49 (12):1767-1772.

Mulvey, J. M., R. J. Vanderbei, and S. A. Zenios. 1995. "Robust optimization of large-scale systems." Operations research 43 (2):264-281. 
Narayanan, S. 2015. "Food security in India: the imperative and its challenges." Asia \& the Pacific Policy Studies 2 (1):197-209.

Nickel, S., F. Saldanha-da-Gama, and H. P. Ziegler. 2012. "A multi-stage stochastic supply network design problem with financial decisions and risk management." Omega 40 (5):511-524.

Önüt, S., U. R.Tuzkaya, and B. Doğaç. 2008. "A particle swarm optimization algorithm for the multiple-level warehouse layout design problem." Computers \& Industrial Engineering 54(4):783-799.

Önüt, S., U. R.Tuzkaya, and B. Doğaç. 2008. “A particle swarm optimization algorithm for the multiple-level warehouse layout design problem." Computers \& Industrial Engineering 54(4):783-799.

Parikh, J., M. Panda, A. Ganesh-Kumar, and V. Singh. 2009. "CO2 emissions structure of Indian economy." Energy34 (8): 1024-1031.

Parvaresh, F., S. M. Husseini, S. H. Golpayegany, and B. Karimi. 2014. "Hub network design problem in the presence of disruptions." Journal of Intelligent Manufacturing 25(4): 755774.

Rahmani, D., R. Ramezanian, P. Fattahi, and M. Heydari. 2013. “A robust optimization model for multi-product two-stage capacitated production planning under uncertainty." Applied Mathematical Modelling 37 (20-21): 8957-8971.

Ramachandra, T. V., Shwetmala. 2009. "Emissions from India's transport sector: Statewise synthesis.” Atmospheric Environment 43 (34): 5510-5517. 
Schipper, L, F. Herbert, and J. Leather. 2009. "Transport and Carbon Dioxide Emissions:Forecasts, Options Analysis, and Evaluation.” Asian Development Bank 9.

Shu, J., Q. Ma, and S. Li. 2010. "Integrated location and two-echelon inventory network design under uncertainty." Annals of Operations Research 181(1): 233-247.

Rosa, D. V., M. Gebhard, E. Hartmann, and J. Wollenweber. 2013. "Robust sustainable bidirectional logistics network design under uncertainty." International Journal of Production Economics 145(1): 184-198.

Thangaraj, R., M. Pant, A. Abraham, and P. Bouvry. 2011. Particle swarm optimization: hybridization perspectives and experimental illustrations. Applied Mathematics and Computation 217(12): 5208-5226.

Wagner, S.M. and C. Bode. 2008. "An empirical examination of supply chain performance along several dimensions of risk.” Journal of business logistics 29 (1):307-325.

Wakeland, W., S. Cholette, and K. Venkat. 2012. Food transportation issues and reducing carbon footprint. In Green technologies in food production and processing (pp. 211-236). Springer, Boston, MA.

Williams, A., S. Kennedy, F. Philipp, and G. Whiteman. 2017. "Systems thinking: A review of sustainability management research.” Journal of Cleaner Production 148: 866-881.

Xin, B., J. Chen, J. Zhang, H. Fang, and Z. H. Peng. 2012. Hybridizing differential evolution and particle swarm optimization to design powerful optimizers: a review and taxonomy. IEEE Transactions on Systems, Man, and Cybernetics, Part C (Applications and Reviews) 42 (5): 744-767. 
Zeballos, L.J., C. A. Méndez, A. P. Barbosa-Povoa, and A. Q. Novais. 2014. "Multi-period design and planning of closed-loop supply chains with uncertain supply and demand." Computers \& Chemical Engineering 66: 151-164.

Zhou, G. D., T. H. Yi, H. Zhang, and H. N. Li. 2015. "A comparative study of genetic and firefly algorithms for sensor placement in structural health monitoring." Shock and Vibration 110.

Zhu, Z., F. Chu, A. Dolgui, C. Chu, W. Zhou, and S. Piramuthu. 2018. "Recent advances and opportunities in sustainable food supply chain: a model-oriented review." International Journal of Production Research 1-23.

Zorya, S., N. Morgan, L. Diaz Rios, R. Hodges, B. Bennett, T. Stathers, P. Mwebaze, and J. Lamb. 2011. "Missing food: the case of postharvest grain losses in sub-Saharan Africa."

Report of the Comptroller and Auditor General of India on Storage Management and Movement of Food Grains in Food Corporation of India. 2013. Union Government Ministry of Consumer Affairs, Food and Public Distribution 